\title{
Simultaneous Cyclic Scheduling and Optimal Control of Multi-Grade Polymerization Reactors
}

Sebastian Terrazas-Moreno, Antonio Flores-Tlacuahuac*

Departamento de Ingeniería y Ciencias Químicas, Universidad Iberoamericana

Prolongación Paseo de la Reforma 880, México D.F., 01210, México

Ignacio E. Grossmann

Department of Chemical Engineering, Carnegie-Mellon University

5000 Forbes Av., Pittsburgh 15213, PA

June 11, 2006

${ }^{*}$ Author to whom correspondence should be addressed. E-mail: antonio.flores@uia.mx, phone/fax: +52(55)59504074, http://200.13.98.241/ antonio 


\begin{abstract}
This paper presents the simultaneous solution of the optimal sequencing and optimal dynamic transitions of two multigrade polymerization CSTRs. The simultaneous formulation results in a Mixed-Integer Dynamic Optimization (MIDO) problem. The profiles of the state variables during dynamic transitions are discretized using orthogonal collocation on finite elements, transforming the MIDO problem into a Mixed-Integer Non Linear Problem. The objective of this formulation is to maximize the profit of the manufacturing operation, considering polymer sales, inventory cost and transition cost. The transition cost term is determined by the duration of the transition stages and the manipulated variable profile during transitions. The two industrial reaction systems are used to analyze the optimal solution and compare it to other solutions obtained using different methods. Finally a sensitivity analysis is carried out to describe the impact of changes in certain problem parameters.
\end{abstract}




\section{Introduction}

The importance of industrial production scheduling and control is broadly recognized. Works on the subject are numerous, focusing on batch as well as continuous production facilities. There has been a recent interest in addressing simultaneous scheduling and control problems [1], [2], [3], [4]. Because scheduling and control (SC) problems have strong interactions it looks reasonable to consider them in a simultaneous and integrated framework.

However, until recent, scheduling and control problems were approached independently. As a matter of fact, scheduling problems commonly assume constant transition times and almost any aspect related to process dynamics. On the other hand, process control problems normally assume fixed production sequences. The close relationship between scheduling and control problems and the importance of a simultaneous solution is currently recognized [1],[2],[3]. Moreover, a recent publication [4] concluded that not including process dynamics in scheduling problem formulations can lead to suboptimal solutions.

An important area of application for a simultaneous scheduling and control approach is the polymer industry. It is now common for polymerization plants to operate in a continuous manner while several product grades are produced using the same equipment. In this context grades are understood as products made from the same polymer but that exhibit different end use properties such as brightness, color, mechanical strength [5]. These end use properties of grades are dependent on molecular weight distribution and monomer conversion, which in turn are determined by operating conditions [6]. Transitions times in grade polymerization systems can be long, resulting in considerable amount of off-spec product which clearly impacts the profitability of plant operation. As such, the choice of transitions to be made during a production sequence is an important aspect to consider when determining a production schedule for polymerization plants. The rigorous inclusion of process dynamics for optimal grade transitions in a scheduling formulation, as proposed in this work, results in a Mixed Integer Dynamic Optimization (MIDO) formulation. 
Nystrom et.al. [3] recently addressed scheduling and grade transition for polymerization systems. The authors proposed a solution strategy for solving MIDO problems based on a decomposition scheme by Barton and Allgor [7]. The MIDO formulation is decomposed in a primal problem containing the dynamic part and a master problem containing the scheduling formulation. Results of this study include a successful treatment of grade transitions during production scheduling for a polymerization plant. However, it should be noted that their strategy is highly application specific. Another approach for addressing the integrated scheduling and control problem has been proposed by Prata et.al. [8]. The dynamic optimization (control) problem is solved using single and multiple shooting methods instead of simultaneous dynamic optimization (SDO) used here. In [8] there is no inventory cost included in the objective function. Instead, the total amount of raw material consumed during the total production schedule is accounted for. Also due to confidentiality reasons the mathematical model of the polymerization process is not available. Therefore, the extent and degree of nonlinearities cannot be known with accuracy.

In our previous work [9] we considered an iterative scheme for simultaneous scheduling and control in which transition times were approximated manually at each iteration. In contrast in this paper we consider explicitly the transition times as part of the mathematical formulation, thereby eliminating the need for the approximate iterative scheme. The MIDO formulation is applied to polymerization reactors which approximate real life applications as opposed to the simpler cases used in previous works to test our former iterative formulation [9].

In the present work a simultaneous cyclic scheduling and optimal control approach is proposed and studied in two polymerization systems. The resulting problem is cast as a Mixed-Integer Dynamic Optimization (MIDO) problem that involves integer variables for sequencing decisions and continuous variables for production and transition times, cycle duration and inventory levels. The solution methodology used in this work [9], consists in transforming the MIDO problem into a MINLP. This method involves the transformation 
of the ordinary differential equations that describe the dynamic model into a set of algebraic equations through the use of orthogonal collocation equations [10]. The problem can then be solved using any known MINLP techniques such as the Outer-Approximation method [11],[12]. This paper emphasizes the analysis of the optimal solutions. One main concern addressed in the following pages is the influence of transition times and profiles on the optimal solution. Both, transition times and profile, are consequences of process dynamics, so their influence on the solution reflects the importance of including rigorous dynamics on the scheduling problems.

\section{Problem definition}

In polymerization plants a certain number of polymer grades are specified in terms of their conversion and/or molecular weight distribution (MWD). These grades are to be formed using a single CSTR operating isothermally. Each grade is obtained from the same raw material but using different operating conditions. Since the reactor operates continuously, the switch from a certain grade to another involves a dynamic transition, which is carried out by manipulating the monomer feed flow rate or the initiator feed flow rate. The transition is accomplished once the conversion and/or MWD are within a certain tolerance of their desired steady state value.

In terms of the scheduling problem, lower bounds for the demands rates are specified for all different polymer grades. In order to satisfy these demands, all grades must be manufactured once during a production cycle whose cyclic time is to be determined. The manufacturing operation involves inventory holding costs and transition costs. Inventory holding costs, raw material costs and product prices of each grade are known, as well as steady state and upper and lower bounds for all variable.

Given the above stated problem the objective of the present work is to find the optimal manufacturing cycle in order to meet all demands for the grades while maximizing profit. The optimal cycle is described by the following decision variables. (1) Grade manufacturing sequence. The sequencing of grades will influence the transitions to be carried 
out. (2) Optimal transition times. Transition costs are functions of transition times and manipulated variable profiles. The duration of those transitions must be calculated so that the switch from one grade to another is carried successfully while minimizing costs. Transition times also influence overall cycle time. (3) Optimal dynamic transitions. The behavior of the manipulated variables during grade transition must be defined in order to minimize transition costs. (4) Optimal cycle duration. The cycle time, which dictates the inventory requirements, must be calculated as to maximize hourly profits. (5) The length of the production runs must be such that the demands are satisfied, while profit is maximized through the production of a profitable grade mix.

Certain assumptions are made in order to obtain the optimal solution: (a) Demand, inventory costs and raw material costs are deterministic parameters, (b) All polymer produced is sold. There is no upper bound on production, (c) All grades are produced only once during the production cycle, (d) Once a grade has been produced it is stored and

depleted until the end of the cycle, (e) Profit is defined as product sales minus inventory holding costs minus transition costs divided by total cycle time (hourly profits). All other costs are not taken into account, (f) Transition cost is defined as the cost of the raw material spent during transition when no useful end product is being manufactured.

\section{Scheduling and Control MIDO Formulation}

As mentioned before, the manufacturing operation relevant to this work is carried out in production cycles. As shown in Figure 1(a) the cyclic time is divided into a series of slots. Within each slot two operations are carried out: (a) the production period during which a given product is manufactured around steady-state conditions and (b) the transition period during which dynamic transitions between two products take place. According to this description, Figure 1(b) depicts a typical dynamic operating response curve within each slot. At the beginning of each slot, material of a given product is manufactured until the demand imposed on such product is met. During this period both the system states $x$ and the manipulated variables $u$ remain constant. Afterwards, the CSTR process 
conditions are changed (by modifying the manipulated variables $u$ ) until new desired process operating conditions (as represented by the system states $x$ ) are reached leading to the manufacture of a new product. In this work we assume that only one product can be produced in a slot and that each product is produced only once within each production wheel. Also, we assume that once a production wheel is completed, new identical cycles are executed indefinitely.

The indices, decision variables and system parameters used in the SSC MIDO problem formulation are as follows:

\section{Indices}

$\begin{array}{ll}\text { Products } & i, p=1, \ldots N_{p} \\ \text { Slots } & k=1, \ldots N_{s} \\ \text { Finite elements } & f=1, \ldots N_{f e} \\ \text { Collocation points } & c, l=1, \ldots N_{c p} \\ \text { System states } & n=1, \ldots N_{x} \\ \text { Manipulated variables } & m=1, \ldots N_{u}\end{array}$


2. Decision variables

$y_{i k} \quad$ Binary variable to denote if product $i$ is assigned to slot $k$

$p_{k} \quad$ Processing time at slot $k$

$t_{k}^{e} \quad$ Final time at slot $k$

$t_{k}^{s} \quad$ Start time at slot $k$

$G_{i} \quad$ Production rate

$T_{c} \quad$ Cyclic time $[\mathrm{h}]$

$x_{f c k}^{n} \quad$ N-th system state in finite element $f$ and collocation point $c$ of slot $k$

$u_{f c k}^{m} \quad$ M-th manipulated variable in finite element $f$ and

collocation point $c$ of slot $k$

$W_{i} \quad$ Amount produced of each product $[\mathrm{kg}]$

$\theta_{i k} \quad$ Processing time of product $i$ in slot $k$

$\theta_{k}^{t} \quad$ Transition time at slot $k$

$\Theta_{i} \quad$ Total processing time of product $i$

$x_{o, f k}^{n} \quad n$-th state value at the beginning of the finite element $f$ of slot $k$

$\bar{x}_{k}^{n} \quad$ Desired value of the $n$-th state at the end of slot $k$

$\bar{u}_{k}^{m} \quad$ Desired value of the $m$-th manipulated variable at the end of slot $k$

$x_{i n, k}^{n} \quad n$-th state value at the beginning of slot $k$

$u_{i n, k}^{n} \quad m$-th manipulated variable value at the beginning of slot $k$

$X_{f c k} \quad$ Conversion in finite element $f$ and collocation point $c$ of slot $k$

$M W D_{f c k} \quad$ Molecular Weight Distribution in finite element $f$

and collocation point $c$ of slot $k$

\section{Parameters}

$N_{p} \quad$ Number of products

$N_{s} \quad$ Number of slots

$N_{f e} \quad$ Number of finite elements

$N_{c p} \quad$ Number of collocation points

$N_{x} \quad$ Number of system states

$N_{u} \quad$ Number of manipulated variables 


\begin{tabular}{|c|c|}
\hline$D_{i}$ & Demand rate $[\mathrm{kg} / \mathrm{h}]$ \\
\hline$C_{i}^{p}$ & Price of products $[\$ / \mathrm{kg}]$ \\
\hline$C_{i}^{s}$ & Cost of inventory $[\$ / \mathrm{kg}-\mathrm{hr}]$ \\
\hline$C^{r}$ & Cost of raw material [\$/lt of feed solution] \\
\hline$C^{I}$ & Cost of initiator $[\$ /$ lt of feed solution $]$ \\
\hline$h_{f k}$ & Length of finite element $f$ in slot $k$ \\
\hline$\Omega_{c c}$ & Matrix of Radau quadrature weights \\
\hline $\bar{x}_{k}^{n}$ & Desired value of the $n$-th system state at slot $k$ \\
\hline $\bar{u}_{k}^{m}$ & Desired value of the $m$-th manipulated variable at slot $k$ \\
\hline$\theta^{\max }$ & Upper bound on processing time \\
\hline$t_{i p}^{t}$ & Estimated value of the transition time between product $i$ and $p$ \\
\hline$x_{s s, i}^{n}$ & $n$-th state steady value of product $i$ \\
\hline$u_{s s, i}^{m}$ & $m$-th manipulated variable value of product $i$ \\
\hline$F_{i}^{o}$ & Feed stream volumetric flow rate at steady state for grade $i$ \\
\hline$M W_{\text {monomer }}$ & Monomer molecular weight $[\mathrm{kg} / \mathrm{kmol}]$ \\
\hline$X_{s s, i}$ & Desired conversion degree of grade $i$ \\
\hline$M W D_{s s, i}$ & Desired molecular weight distribution of grade $i$ \\
\hline$x_{\min }^{n}, x_{\max }^{n}$ & Minimum and maximum value of the state $x^{n}$ \\
\hline$u_{\min }^{m}, u_{\max }^{m}$ & Minimum and maximum value of the manipulated variable $u^{m}$ \\
\hline$\dot{x}_{t o l}^{n}$ & Maximum absolute value for state derivatives at the end of dynamic transition \\
\hline$x_{t o l}^{n}$ & $\begin{array}{l}\text { Maximum absolute deviation from desired final value, allowable for state } \\
\text { variable } x^{n} \text { at the end of dynamic transition }\end{array}$ \\
\hline$u_{\text {cont }}^{f}$ & Maximum absolute change for $u^{m}$ between finite elements \\
\hline$u_{\text {cont }}^{c}$ & Maximum absolute change for $u^{m}$ between collocation points \\
\hline$\gamma_{c}$ & Roots of the Lagrange orthogonal polynomial \\
\hline
\end{tabular}

In order to clarify the SSC MIDO problem formulation, it has been divided into two parts. The first one deals with the scheduling part and the second one with the dynamic optimization part. 


\section{- Objective function.}

$$
\begin{aligned}
\max & \left\{\sum_{i=1}^{N_{p}} \frac{C_{i}^{p} W_{i} / T c}{T_{c}}-\sum_{i=1}^{N_{p}} \frac{C_{i}^{s}\left(G_{i}-W_{i} / T_{c}\right) \theta_{i}}{2}\right. \\
& \left.-\left[\sum_{k=1}^{N_{s}} \sum_{f=1}^{N_{f} e} h_{f c k} \theta_{k}^{t} Q_{\max }^{m} \sum_{c=1}^{N_{c p}} u_{f c k}^{m} \gamma_{c}\right] \frac{C^{r}}{T_{c}}-\sum_{k=1}^{N_{s}} Q_{\max }^{I} u^{I} \theta_{k}^{t} \frac{C^{I}}{T_{c}}\right\}
\end{aligned}
$$

The total process profit is given by the amount and cost of the manufactured products minus the sum of the inventory costs and the product transition costs. The first term of the objective function represents product sales. The second term states that inventory costs are calculated from the amount to be stored of each grade, and the time this production must be hold (from midpoint through the production stage until the end of the cycle). The third and fourth terms refer to transition costs in terms of wasted, offspec material during transitions. The offspec material is calculated as the amount of raw material and initiator fed into the reactor for all the duration of the transition. Since monomer flow

rate is a manipulated variable, the term $\sum_{c=1}^{N_{c p}} u_{f c k}^{m} \gamma_{c}$ represents a weighted average of the manipulated variable for each finite element. This type of transition cost forces the transition to be done as economically as possible, which usually derives in fast transitions in order to waste the least amount of raw material.

1. Scheduling part.

a) Product assignment

$$
\begin{aligned}
& \sum_{k=1}^{N_{s}} y_{i k}=1, \forall i \\
& \sum_{i=1}^{N_{p}} y_{i k}=1, \forall k
\end{aligned}
$$

Equation 2a states that, within each production wheel, any product can only be manufactured once, while constraint $2 \mathrm{~b}$ implies that only one product is 
manufactured at each slot. Due to this constraint, the number of products and slots turns out to be the same.

b) Amounts manufactured

$$
\begin{aligned}
& W_{i} \geqslant D_{i} T_{c}, \forall i \\
& W_{i}=G_{i} \Theta_{i}, \forall i \\
& G_{i}=F_{i}^{o} C_{m 0} M W_{\text {monomer }}, \forall i
\end{aligned}
$$

Equation 3a states that the total amount manufactured of each product $i$ must be equal or greater than the specified demand rate times the duration of the production wheel, while Equation 3b indicates that the amount manufactured of product $i$ is computed as the product of the production rate $\left(G_{i}\right)$ times the time used $\left(\Theta_{i}\right)$ for manufacturing such product. The production rate is computed from Equation 3c as a simple relationship between the feed stream flowrate $\left(F_{i}^{o}\right)$, the feed stream composition $\left(C_{m 0}\right)$, and monomer molecular weight $\left(M W_{\text {monomer }}\right)$.

c) Processing times

$$
\begin{aligned}
\theta_{i k} & \leqslant \theta^{\max } y_{i k}, \forall i, k \\
\Theta_{i} & =\sum_{k=1}^{N_{s}} \theta_{i k}, \forall i \\
p_{k} & =\sum_{i=1}^{N_{p}} \theta_{i k}, \forall k
\end{aligned}
$$

The constraint given by Equation 4a sets an upper bound on the time used for manufacturing product $i$ at slot $k$. Equation $4 \mathrm{~b}$ is the time used for manufacturing product $i$, while Equation $4 \mathrm{c}$ defines the duration time at slot $k$. 
e) Timing relations

$$
\begin{aligned}
t_{k}^{e} & =t_{k}^{s}+p_{k}+\theta_{k}^{t}, \forall k \\
t_{k}^{s} & =t_{k-1}^{e}, \forall k \neq 1 \\
t_{k}^{e} & \leqslant T_{c}, \forall k \\
t_{f c k} & =(f-1) \frac{\theta_{k}^{t}}{N_{f e}}+\frac{\theta_{k}^{t}}{N_{f e}} \gamma_{c}, \forall f, c, k
\end{aligned}
$$

Equation 5a is used for computing the time at the end of each slot as the sum of the slot start time plus the processing time and the transition time. Equation 5b states that the start time of all the slots, different than the first one, is just the end time of the previous slot. Equation $5 \mathrm{c}$ is used to force that the end time at each slot be less than the production wheel cyclic time. Finally, Equation 5d is used to obtain the time value inside each finite element and for each internal collocation point.

\section{Dynamic Optimization part.}

To address the optimal control part, the simultaneous approach [10] for solving dynamic optimization problems was used. In this approach the dynamic mathematical model representing system behavior is discretized using the method of orthogonal collocation on finite elements [13],[14]. According to this procedure, a given slot $k$ is divided into a number of finite elements. Within each finite element an adequate number of internal collocation points is selected as depicted in Figure 2. Using several finite elements is useful to represent dynamic profiles with non-smooth variations. Thereby, the set of ordinary differential equations comprising the system model, is approximated at each collocation point leading to a set of nonlinear equations that must be satisfied. 
a) Dynamic mathematical model discretization

$$
x_{f c k}^{n}=x_{o, f k}^{n}+\theta_{k}^{t} h_{f k} \sum_{l=1}^{N_{c p}} \Omega_{l c} \dot{x}_{f l k}^{n}, \forall n, f, c, k
$$

The constraints given by Equations 6 are used to compute the value of the system states at each one of the discretized points $\left(x_{f c k}^{n}\right)$ by using the monomial basis representation. $x_{o, f k}^{n}$ is the $n$-th system state at the beginning of each element, $\Omega_{l c}$ is the collocation matrix and $\dot{x}_{f c k}^{n}$ is the first order derivative of the $n$-th state. Notice that when working with the first element, $x_{o, 1 k}^{n}$ represents the specified initial value of the $n$-th state. Also notice that in the present formulation the length of all finite elements is the same and computed as

$$
h_{f k}=\frac{1}{N_{f e}}
$$

b) Continuity constraint between finite elements

$$
x_{o, f k}^{n}=x_{o, f-1, k}^{n}+\theta_{k}^{t} h_{f-1, k} \sum_{l=1}^{N_{c p}} \Omega_{l, N_{c p}} \dot{x}_{f-1, l, k}^{n}, \forall n, f \geqslant 2, k
$$

In the simultaneous approach for dynamic optimization problems, the states must be continuous when crossing from one given finite element to the next one. We use Equations 8 to force continuous state profiles on all the elements at the beginning of each element $\left(x_{o, f k}^{n}\right)$ and they are computed in terms of the same monomial basis used before for defining the value of the system states.

c) Model behavior at each collocation point

$$
\dot{x}_{f c k}^{n}=f^{n}\left(x_{f c k}^{1}, \ldots, x_{f c k}^{n}, u_{f c k}^{1}, \ldots u_{f c k}^{m}\right), \forall n, f, c, k
$$

Equations 9 are used for computing the value of the first order derivatives of the systems at finite element $f$ of collocation point $c$ in slot $k$. Those equations 
simply represent the right hand sides of the dynamic model. Because our scheduling and control formulation is system independent, we have used the notation $f^{n}$ to represent the right hand side of the $n$-th ordinary differential equation describing any desired dynamic system.

d) Initial and final controlled and manipulated variable values at each slot:

$$
\begin{aligned}
& x_{i n, k}^{n}=\sum_{i=1}^{N_{p}} x_{s s, i}^{n} y_{i, k}, \forall n, k \\
& \bar{x}_{k}^{n}=\sum_{i=1}^{N_{p}} x_{s s, i}^{n} y_{i, k+1}, \forall n, k \neq N_{s} \\
& \bar{x}_{k}^{n}=\sum_{i=1}^{N_{p}} x_{s s, i}^{n} y_{i, 1}, \forall n, k=N_{s} \\
& u_{i n, k}^{m}=\sum_{i=1}^{N_{p}} u_{s s, i}^{m} y_{i, k}, \forall m, k \\
& \bar{u}_{k}^{m}=\sum_{i=1}^{N_{p}} u_{s s, i}^{m} y_{i, k+1}, \forall m, k \neq N_{s}-1 \\
& \bar{u}_{k}^{m}=\sum_{i=1}^{N_{p}} u_{s s, i}^{m} y_{i, 1}, \forall m, k=N_{s} \\
& x_{N_{f e}, N_{c p}, k}^{n}=\bar{x}_{k}^{n}, \forall n, k \\
& u_{1,1, k}^{m}=u_{i n, k}^{m}, \forall m, k \\
& u_{N_{f e}, N_{c p}, k}^{m}=\bar{u}_{i n, k}^{m}, \forall m, k \\
& x_{o, 1, k}^{n}=x_{i n, k}^{n}, \forall n, k \\
& x_{t o l, k}^{n} \geqslant x_{N f e, N c, k}^{n}-\bar{x}_{k}^{n}, \forall n, k \\
& -x_{t o l, k}^{n} \leqslant x_{N f e, N c, k}^{n}-\bar{x}_{k}^{n}, \forall n, k
\end{aligned}
$$

Equations 10 define the values of the state variables at the beginning of each slot $k\left(x_{i n, k}^{n}\right)$. The desired value of each state at the end of the same slot $k\left(\bar{x}_{k}^{n}\right)$ is computed in Equations 11-12. It should be stressed that the state values at the beginning and end of each slot $k$ are given by the corresponding steadystate values $\left(x_{s s, i}^{n}\right)$ calculated a priori. $x_{s s, i}^{n}$ simply stands for the steady-state 
value of the manufacturing product $i$. They can be easily obtained from openloop steady-state simulation of the processing system. Similarly, Equations 13-15 define the values of the manipulated variables at the beginning of each slot $k\left(u_{i n, k}^{m}\right)$ and at the end of the slot $k\left(\bar{u}_{k}^{m}\right)$. Equations 16 enforce the system states to take the desired state values at each slot $k$. A similar situation occurs with the values of the manipulated variables. Equations 17 fix the values at the first finite element and first collocation point of each slot $k\left(u_{1,1, k}^{m}\right)$ as the value that such variable takes at the beginning of the same slot $k$. Equations 18 determine the values of the manipulated variables at the last finite element and last collocation point of slot $k\left(u_{N_{f e}, N_{c p}, k}^{m}\right)$ as the desired steady-state value of the same variable at slot $k\left(\bar{u}_{k}^{m}\right)$. Equations 19 determine the values of the system states at the beginning of each slot $\left(x_{o, 1, k}^{n}\right)$. Finally, equations 20 and 21 set the allowable deviation from desired state variable values at the end of every dynamic transition.

e) Lower and upper bounds on the decision variables

$$
\begin{aligned}
& x_{\text {min }}^{n} \leqslant x_{f c k}^{n} \leqslant x_{\text {max }}^{n}, \forall n, f, c, k \\
& u_{\text {min }}^{m} \leqslant u_{f c k}^{m} \leqslant u_{\text {max }}^{m}, \forall m, f, c, k
\end{aligned}
$$

Equations 22a-22b simply constrain the values of both the system states and manipulated variables to lie within acceptable lower and upper bounds. 
f) Smooth transition constraints

$$
\begin{aligned}
& u_{f, c, k}^{m}-u_{f, c-1, k}^{m} \leqslant u_{\text {cont }}^{c}, \forall m, k, c \neq 1 \\
& u_{f, c, k}^{m}-u_{f, c-1, k}^{m} \geqslant-u_{\text {cont }}^{c}, \forall m, k, f, c \neq 1 \\
& u_{f, 1, k}^{m}-u_{f-1, N f e, k}^{m} \leqslant u_{\text {cont }}^{f}, \forall m, k, f \neq 1 \\
& u_{f, 1, k}^{m}-u_{f-1, N f e, k}^{m} \geqslant-u_{\text {cont }}^{f}, \forall m, k, f \neq 1 \\
& u_{1,1, k}^{m}-u_{i n, k}^{m} \leqslant u_{\text {cont }}^{f}, \forall k \\
& u_{1,1, k}^{m}-u_{i n, k}^{m} \geqslant-u_{\text {cont }}^{f}, \forall k \\
& \dot{x}_{N f e, N c p, k} \geqslant-\dot{x}_{\text {tol }, k}, \forall n, k \\
& \dot{x}_{N f e, N c p, k} \leqslant \dot{x}_{\text {tol }, k}, \forall n, k
\end{aligned}
$$

Equations 8 are used to ensure continuity between adjacent finite elements. However no equation up to this point forces a smooth profile for the manipulated variable. Equations 23- 28 force the change between adjacent collocation points and finite elements to be within a certain acceptable range. Equations 29 and 30 are used to make sure that at the end of the dynamic transition the system is at, or very close to, steady state conditions.

\section{Case Studies}

In order to analyze the performance of the simultaneous scheduling and control formulation presented in this paper, it was tested on two polymerization processes. In the following section these processes are described and the results of the scheduling and control problem are discussed. The models used are intended to resemble industrial processes, but they are still simplified versions of complex models used in industrial applications. 


\subsection{Case 1: High Impact Polystyrene (HIPS)}

\subsubsection{Process description}

The isothermal free radical bulk polymerization of styrene was carried out in a CSTR. The single CSTR model has been used in previous works $[15,16,6]$ to describe this process. In the following example the model is cast in dimensionless form by diving each state variable and manipulated variable by its maximum values. Design information and scaling values are given in Table 1.

$$
\begin{aligned}
& \frac{d x_{1}}{d t}=\frac{Q_{\text {imax }} x_{1 o} u_{i}-Q_{\max } x_{1} u_{f}}{V}-k_{d} x_{1} \\
& \frac{d x_{2}}{d t}=Q_{\max } u_{f} \frac{x_{2 o}-x_{2}}{V}-k_{p} x_{2}\left(\mu_{\max }^{r} x_{6}+\mu_{b \max }^{0} x_{7}\right) \\
& \frac{d x_{3}}{d t}=Q_{\max } u_{f} \frac{x_{3 o}-x_{3}}{V}-x_{3}\left(k_{I 2} C_{r \max } x_{4}+k_{f s} \mu_{r \max }^{0} x_{6}+k_{f b} \mu_{b \max }^{0} x_{7}\right) \\
& \frac{d x_{4}}{d t}=2 f_{a} \frac{k_{d} C_{\text {imax }} x_{1}}{C_{\text {rmax }}}-x_{4}\left(k_{I 1} C_{\max } x_{2}+k_{I 2} C_{\text {bmax }} x_{3}\right) \\
& \frac{d x_{5}}{d t}=\frac{C_{b \max } x_{3}}{C_{b r \max }}\left(k_{I 2} C_{r \max } x_{4}+k_{f b}\left(\mu_{r \max }^{0} x_{6}+\mu_{b \max }^{0} x_{7}\right)\right)-x_{5}\left(k_{I 3} C_{\max } x_{2}+k_{t}\right. \\
& \left.\left(\mu_{r \max }^{0} x_{6}+\mu_{b \max }^{0} x_{7}+C_{b \max } x_{5}\right)\right) \\
& \frac{d x_{6}}{d t}=\frac{2 k_{I 0}\left(C_{\max } x_{2}\right)^{2}+k_{I 1} C_{r \max } x_{4} C_{\max } x_{2}+C_{\max } x_{2} k_{f s} \mu_{r \max }^{0} x_{6} \mu_{b \max }^{0} x_{7}}{\mu_{r \max }^{0}} \\
& -\left(k_{p} C_{\text {mmax }} x_{2}+k_{t}\left(\mu_{r \max }^{0} x_{6}+\mu_{\text {bmax }}^{0} x_{7}+C_{\text {brmax }} x_{5}\right)+k_{f s} C_{\text {mmax }} x_{2}\right. \\
& \left.+k_{f b} C_{b \max } x_{3}\right) x_{6}+k_{p} C_{\max } x_{2} x_{6} \\
& \frac{d x_{7}}{d t}=\frac{k_{I 3} C_{b r \max } C_{\max } x_{5} x_{2}}{\mu_{b \max }^{0}}-\left(k_{p} C_{\text {mmax }} x_{2}+k_{t}\left(\mu_{r \max }^{0} x_{6}+\mu_{b \max }^{0} x_{7}+C_{b r \max } x_{5}\right)\right. \\
& \left.+k_{f s} C_{\text {mmax }} x_{2} k_{f b} C_{b \max } x_{3}\right) x_{7}+k_{p} C_{\max } x_{2} x_{7}
\end{aligned}
$$

where

$$
\begin{aligned}
& x_{i}=(\text { Value of state } i) /(\text { Max expected value of state } i) \\
& \left.u_{i}=\text { (Value of initiator flow }\right) / \text { (Max expected value of initiator flow) } \\
& \left.u_{f}=\text { (Value of feed stream flow }\right) / \text { (Max expected value of feed stream flow) }
\end{aligned}
$$

Five different HIPS grades $(A, B, C, D, E)$ were defined as desired products for the 
production cycle, corresponding to 15, 25, 35, 40 and 45 percent monomer conversion. Due to gel effect, normally higher conversions are not obtained in a single reactor. Instead, a sequence of CSTRs is used for that purpose. Monomer flow rate $\left(Q_{m}\right)$ was chosen as the manipulated variable during grade transitions. Detailed grade information is shown in Table 2.

As described in previous sections, the simultaneous scheduling and control formulation yields a MIDO problem, which is transformed into a MINLP using the SDO technique with 20 finite elements and three collocation points. The problem is solved in GAMS

using DICOPT, a MINLP solver that uses the outer approximation algorithm [11],[12]. The formulation consists of 6666 constraints, 6267 continuous variables and 25 discrete variables. The solution took $7640 \mathrm{~s}$ of cpu time using an IBM Laptop $1.6 \mathrm{GHz}$ processor and 256 MB RAM. Results of the optimal production cycle, $E \rightarrow A \rightarrow B \rightarrow C \rightarrow D$ with objective $\$ 1456 /$ hr and length of cycle time $32.3 \mathrm{~h}$, are shown in Figure 3 and Table 3 .

In the following section the key drivers for the optimal solution are first discussed, in a second part a dynamic analysis of the transitions is included, and in a third part a comparison versus a sequential solution method is carried out. Finally, the present formulation will be compared with an earlier MIDO version for simultaneous scheduling and control proposed by our research group [9]. In the sequential solution optimal dynamic profiles for every possible transition are calculated in a first step and introduced as fixed parameters for the scheduling problem.

\section{Optimal solution analysis}

In order to understand the drivers for maximum profit, the optimal and five other feasible solutions (as found by DICOPT/CONOPT3) were analyzed . The main variable level and some arbitrary indicators used mainly for comparison among solutions are reported in Table 4 and Figure 4. The following key observations can be made. (a) Since 
product $D$ is one of the most profitable products (second highest price, low inventory costs), the optimizer allocates the largest portion of time to the production of this grade, while just satisfying the demand for all other grades. (b) Transition times affect the results of each feasible solution in two ways. First, by affecting total cycle time and second by affecting transition costs. In general lower transition times relate to lower transition time to total cycle time ratio and lower transition costs. (c) Determining total cycle time involves a trade-off between transition and inventory costs. If the total cycle time is short, the inventory costs are low but transition times take up a considerable proportion of the total cycle duration. On the other hand, in the longer cycle times the ratio of transition times to cycle time is low, and productivity of the cycle is high at the expense of incurring in high inventory costs. The magnitude and proportion of costs in the present example is such that a short cycle time with low inventory costs is preferred over a long cycle. Therefore, minimizing transition times becomes very important to avoid the high ratio of transition times to total cycle time.

\section{Dynamic analysis}

Dynamic behavior during each grade transition dictates both transition times and transition costs. Each transition has different dynamic behavior, and it is the objective of this section to understand the influence of this behavior on the selection of the optimal schedule

for cyclic production. In order to carry out this analysis, the system was linearized around each steady state, and all possible grade transitions were characterized by a transfer function. Time constants were then calculated. Since they were found to be proportional to total transition time this last indicator will be used for analysis. Dominant eigenvalues for each linearized steady state are given in Table 5. The real part of all the eigenvalues is negative and their magnitude decreases as grade conversion increases. This makes sense since lower grade conversions are achieved by higher feed stream flow rates, which in turn results in faster dynamic behavior around lower conversion steady states. Initially, the 
optimal schedule features an $E$ to $A$ product transition. This can be explained as the coupling of the slowest operating region (around grade $E$ ) with a faster one (around grade A). This allows all other transitions to be made between grades of adjacent monomer conversion ( $A$ to $B, B$ to $C$ and so on). This kind of transitions take the least amount of effort since the magnitude of the change in the manipulated variable value is minimized.

From the previous paragraph it is unclear why the optimal schedule corresponds to transitions from lower conversion grades (as in $A$ to $B$ ) and not from higher conversion to lower conversion (as in $B$ to $A$ ) since both type of transitions demand the same magnitude of change in the feed stream flow rate. However, the dynamic behavior between the two type of transitions can be expected to be very different. To understand this result, two extra calculations were performed. From dynamic optimization, transition costs and transition times were calculated. Cycles for minimum transition costs and minimum transition times were then calculated using a reduced MILP model with GAMS/CPLEX. The optimal sequence for minimum transition times was $E \rightarrow A \rightarrow B \rightarrow C \rightarrow D$, while the optimal sequence for minimum transition costs was $D \rightarrow C \rightarrow B \rightarrow A \rightarrow E$. Note that the minimum transition time sequence chooses mostly transitions from lower conversion grades to higher conversion grades, while minimum transition cost schedule chooses transitions from higher to lower conversion grades. Another important observation is that the sequence of minimum transition times schedule is exactly the same one as in the schedule chosen for the simultaneous scheduling and control formulation.

Summarizing, reducing total transition times seem to be the main criterion for choosing an optimal sequence. Furthermore, in order to achieve low transition times in the particular system being analyzed, transitions must be made between adjacent grades going from low to high conversion states ( $A$ to $B, B$ to $C$, etc.).

To test the above objective, a modification was performed on the operating parameters of the HIPS polymerization system that consisted in decreasing the reactor vol- 
ume from 6000 to $2500 \mathrm{~L}$, and increasing the initiator flow rate in order to obtain the same grades as in the original system. The optimal grade transition schedule is given by $E \rightarrow A \rightarrow B \rightarrow C \rightarrow D$, the same sequence as the optimal schedule. Comparing the eigenvalues from Table 5, one could expect the overall dynamics to be faster than the one corresponding to the original system. Given this difference, the optimal schedule for the simultaneous scheduling and control problem is exactly the same as in the original case, and once again, it corresponds to the minimum transition time sequence. Differences in the dynamic transitions for the optimal cycle between the original and modified systems can be compared in Figure 5. Because the modified reactor features a smaller residence time, transition between grades are faster.

Given the same prices and costs, the profit is $\$ 1910 / \mathrm{hr}$ for the $2500 \mathrm{~L}$ reactor vs. $\$ 1456 / \mathrm{hr}$ for the $6000 \mathrm{~L}$ reactor, while the cycle time changes from $32.3 \mathrm{hr}$ to $33 \mathrm{hr}$ Since the sequence is the same, the improvement can be attributed solely to faster dynamics for the modified system.

\section{Comparison vs. sequential solution}

We compare the performance of our simultaneous SC formulation compared to a traditional sequential approach. In the sequential approach the SC problems is solved in two parts. The first step consists in calculating all possible transitions between grades as purely dynamic optimization problems, while in the second step this information is used for posing and solving a scheduling problem.

The objective function used for the dynamic optimizations in step 1 of the sequential solution is as follows:

$$
\min \left\{T_{\text {trans }} Q_{\text {mmax }} C r_{m} \sum_{i=1}^{N f e} h_{i}\left(u m_{i, 1} 0.15+u m_{i, 2} 0.50+u m_{i, 1} 0.35\right)+T_{\text {trans }} Q_{i} C r_{i}\right\}
$$

The aim of this objective function is to minimize the transition cost, which is a com- 
bination of the transition time and the feed streams flow rate during the transition. Numerical Results for the sequential solution are found in Table 6; the reader can compare this data with Table 3. A comparison between sales, inventory costs and transition costs is found in Table 7. It is evident that the simultaneous solution produces a better solution than the corresponding one from the sequential approach $(\$ 1456 /$ hr vs. $\$ 1416 / \mathrm{hr})$. The main difference between both approaches is attributed to longer transition times for $\mathrm{E}$ to A transition in the sequential approach (proof on this later). To better understand the difference in dynamic transition profiles for $\mathrm{E}$ to $\mathrm{A}$ transition as determined for the sequential and simultaneous solutions is shown in Figure 6. All other profiles are identical and therefore are not shown. The profile obtained by the simultaneous formulation starts with a more aggressive behavior for the manipulated variable, which in the end allows a shorter transition time, and surprisingly a cheaper transition. Also, in the simultaneous approach the transition time becomes more important since it is involved in other terms in the objective function, which pushes for a faster transition that in turn ends up also being cheaper.

Finally in order to demonstrate that the differences between the simultaneous and sequential results are originated by the longer transition times and higher transitions costs of the $E$ to $A$ transition the following procedure was performed. The transition costs and times obtained by pure dynamic optimization for the transition $E$ to $A$ in the first step of the sequential method were exchanged for the values obtained for the same transition in the simultaneous solution. With these new values the second step in the sequential approach (purely scheduling part) was solved again. The new results are found in Table 8. The performance of the sequential solution is almost as good as the simultaneous solution. This proves that the difference between the original sequential solution and the simultaneous solution was indeed originated by the different dynamic transitions for the $E$ to $A$ transition. 


\section{Comparsion vs. iterative (previous) formulation}

Earlier versions of the simultaneous scheduling and control formulation proposed by our research group [9] are similar to the one proposed in this work. The main differences are: (1) Inventory costs and transition costs terms in the objective function are different, (2) Transition times are calculated differently. In the present work they are calculated directly by the solver, whereas in the previous formulation they had to be fed as fixed parameters estimated from pure dynamic optimization data, and then fine tuned through an iterative process, (3) The earlier formulation fixes transition times as mentioned above. For the optimizer to be able to determine which transition time parameter to use, two extra binary variables are required. These variables will be detailed later.

The first two differences described above require some extra constraints for the present formulation. Since transition times are to be determined by the solver, desired state variable values are within a product quality band given by equation 21 rather than punctual desired value as in the previous formulation. If a tolerable range is not provided then carrying out the transition would take an unreasonably long time. On the other hand, since the square difference of the manipulated variable is not included in the objective function of the present formulation, constraints 25 to 30 are required in the present work.

The following objective function corresponds to the previous formulation [9] applied to the HIPS polymerization system described in this section.

$\begin{aligned} \max & \left\{\sum_{i=1}^{N_{p}} \frac{C_{i}^{p} W_{i}}{T_{c}}-\sum_{i=1}^{N_{p}} \frac{C_{i}^{s}\left(G_{i}-W_{i} / T c\right) \theta_{i}}{2}-\sum_{k=1}^{N_{s}} \sum_{f=1}^{N_{f e}} h_{f k} \sum_{c=1}^{N_{c p}} \frac{C^{r} t_{f c k} \Omega_{c, N_{c p}}}{T_{c}}\left(\left(x_{f c k}^{1}-\bar{x}_{k}^{1}\right)^{2}\right.\right. \\ & \left.\left.+\ldots+\left(x_{f c k}^{n}-\bar{x}_{k}^{n}\right)^{2}+\left(u_{f c k}^{1}-\bar{u}_{k}^{1}\right)^{2}+\ldots+\left(u_{f c k}^{m}-\bar{u}_{k}^{m}\right)^{2}\right)\right\}\end{aligned}$

The reader can compare this equation with equation 1 . Not only is the inventory costs term expressed differently but the transition cost term is defined in a completely different way. Transition costs in equation 39 are expressed as the sum of the square differences of 
every state and the manipulated variable in each discretization point versus the desired value at the end of the transition. The advantage of using this kind of transition term is that smooth transitions are achieved. However, although there is a factor used to convert this sum into money, mathematically converting a square difference of states into money is not very intuitive and its applicability could be questioned.

The extra binary variables required are defined and justified in the following paragraph:

$$
\begin{aligned}
& y_{i, 1}^{\prime}=y_{i, N_{s}}, \forall i \\
& z_{i p k} \geqslant y_{p k}^{\prime}+y_{i k}-1, \forall i, p, k
\end{aligned}
$$

Equation 40 defines backward binary variable $\left(y_{i k}^{\prime}\right)$ meaning that such variable for product $i$ in slot $k$ takes the value assigned to the same binary variable in equation 2 a and $2 \mathrm{~b}$ but one slot backwards $k-1$. At the first slot, Equation 40 defines the backward binary variable as the value of the same variable at the last slot. This type of assignment reflects our assumption of cyclic production wheel. The variable $y_{i k}^{\prime}$ will be used later to determine the sequence of product transitions. The constraint given in Equation 41 is used for defining the binary production transition variable $z_{i p k}$. If such variable is equal to 1 then a dynamic transition will occur from product $i$ to product $p$ within slot $k, z_{i p k}$ will be zero otherwise.

Results for the iterative formulation are found in Table 9 and Figure 7. From a computation demand point of view, the Iterative method features 6121 equations, 6372 continuous variables, 50 binary variables. The solution of this formulation took 11190 cpu s on a 1.6 Ghz processor, while the Direct method features 6666 equations, 6242 continuous variables, 25 binary variables and required $7640 \mathrm{cpu}$ s on the same processor.

Finally, it should be said that the direct formulation requires extra constraints (20 to 30) and the addition of transition times as decision variables increases the non convexity 
of the problem. However, these conditions were found not to be of any significance in terms of computational effort. In fact, the decrease in number of binary variables allowed for an overall faster solution for the direct method. On the other hand the direct formulation is considered to be more realistic and represents an important improvement towards achieving industrial applicability.

\subsection{Case 2: Methyl-Methacrylate Polymerization (MMA)}

A second case study corresponding to the Methyl-Methacrylate Polymerization system is included in the following section. Once the process is described a discussion regarding the optimal solution characteristics, similar to the one performed in the HIPS case study, is carried out. Since the mathematical formulation used is identical to that used in the HIPS example, no comparison versus sequential and iterative procedures is included for this example. At the end of this section a sensitivity analysis is performed in order to understand the effect of changes in process parameters on the optimal solution.

\subsubsection{Process description}

In the following section the bulk free-radical polymerization of Methyl-Methacrylate in an isothermal CSTR is described. The system has already been presented by Congalidis et al. [17] and used by Mahadevan et al. [18] to address grade transition problems from a control point of view. The mathematical model was cast in dimensionless form by dividing each state and flow rates of monomer and initiator by their maximum expected values. 


$$
\begin{aligned}
\frac{d x_{1}}{d t} & =-\frac{V}{\hat{Q}_{m}}\left(k_{p}+k_{f m}\right) x_{1} \sqrt{\frac{2 f^{*} k_{i} \hat{C}_{i} x_{2}}{k_{t d}+k_{t c}}}+\bar{Q}_{m}\left(x_{1_{i n}}-x_{1}\right) \\
\frac{d x_{2}}{d t} & =-\frac{V k_{i}}{\hat{Q}_{m}} x_{2}+\frac{1}{\hat{Q}_{m} \hat{C}_{i}}\left(\hat{Q}_{i} \hat{C}_{i} x_{2_{i n}} \bar{Q}_{i}-\bar{Q}_{m} \hat{Q}_{m} \hat{C}_{i} x_{2}\right) \\
\frac{d x_{3}}{d t} & =\frac{V\left(0.5 k_{t c}+k_{t d}\right)}{\hat{Q}_{m} \hat{D}_{0}}\left[\frac{2 f^{*} k_{i} \hat{C}_{i} x_{2}}{k_{t d}+k_{t c}}\right]+\frac{V k_{f m} \hat{C}_{m}}{\hat{Q}_{m} \hat{D}_{0}} x_{1} \sqrt{\frac{2 f^{*} k_{i} \hat{C}_{i} x_{2}}{k_{t d}+k_{t c}}}-\bar{Q}_{m} x_{3} \\
\frac{d x_{4}}{d t} & =\frac{V M_{m}\left(k_{p}+k_{f m}\right) \hat{C}_{m}}{\hat{Q}_{m} \hat{D}_{1}} x_{1} \sqrt{\frac{2 f^{*} k_{i} \hat{C}_{i} x_{2}}{k_{t d}+k_{t c}}}-\bar{Q}_{m} x_{4} ;
\end{aligned}
$$

where $x_{1}=\frac{C_{m}}{\hat{C}_{m}}, x_{2}=\frac{C_{i}}{\hat{C}_{i}}, x_{3}=\frac{D_{0}}{\hat{D}_{0}}, x_{4}=\frac{D_{1}}{\hat{D}_{1}}, x_{1_{i n}}=\frac{C_{m_{i n}}}{\hat{C}_{m}}, x_{2_{i n}}=\frac{C_{i_{i n}}}{\hat{C}_{i}}, \bar{Q}_{m}=\frac{Q_{m}}{\hat{Q}_{m}}, \bar{Q}_{i}=\frac{Q_{i}}{\hat{Q}_{i}}$. In Table 10 information regarding steady-state design and reactor scaling is presented.

For testing our simultaneous scheduling and control formulation four polymer grades $(A, B, C, D)$ were defined which correspond to molecular weight distributions of 15000 , 25000, 35000 and 45000 . The initiator flow rate $\left(Q_{i}\right)$ was selected as the manipulated variable to achieve grade transition. Table 11 contains the steady-state values of the states and the manipulated variable leading to the manufacture of each one of the $A, B, C$ and $D$ grades. Also shown in Table 11 are the demand rates and costs of each grade.

Once again the resulting MINLP problem was solved using DICOPT/GAMS. The problem consisted of 3325 constraints, 3042 continuous variables and 16 binary variables. Table 12 and figure 8 show the results of solving the scheduling and control problem. The solution took $70 \mathrm{CPU}$ s using a $1.6 \mathrm{GHz}$ processor.

In this section an optimal solution analysis, similar to the one performed for the HIPS case study, is carried out. The findings of the previous case study will be compared against those derived from the optimal solution analysis in the MMA system. Also, a sensitivity analysis including changes in inventory costs, raw material costs, grade quality tolerance, and demand is performed and analyzed.

\section{Optimal solution analysis}


The optimal solution found is compared against four other feasible solutions found by DICOPT, using CONOPT3 as the NLP solver. The same indicators used to compare the different solutions in the HIPS example are used for the MMA case study. These indicators are found in Table 13. A graphical comparison among the different solutions is found in Figure 9.

In the MMA case study the optimal solution and the first three suboptimal solutions have very similar results. However, the optimal solution has a slightly better performance according to the chosen performance indicators. This fact indicates that the measures chosen to evaluate the performance of a particular solution are effective not only for the HIPS problem but also for the present case study.

Note that product $D$ has the highest selling price, and although it has a slightly higher inventory cost than product $C$ (next in selling price) it is the most profitable grade to sell. This is reflected by the fact that the optimizer allocates the longest production time to this grade, resulting in the highest production of all four grades. Just as in the HIPS case study, demand for all other grades is just satisfied while production for grade $D$ exceeds its demand (recall that it is assumed that there is no upper bound on product consumption). Once again the best solution is the one that has the highest ratio of $D$ production as well as production to demand ratio for this grade (see Table 13). In this example transition time is also a key driver for the optimal solution. The best solution is the one with the shortest transition time and the lowest ratio of transition time to total cycle time. This makes sense since the lowest the portion of cycle time devoted to transitions, the highest the available time for profitable production becomes. It is interesting to see that transition costs are actually higher in the optimal solution than in the next best solution. This stresses the fact found in the MMA example that transition times are more important for a profitable operation than transition costs (see transition times and transition costs in Figure 9 and Table 13). Perhaps the only difference in optimal solution behavior between the HIPS and the MMA case studies is the fact that the MMA optimal solution has a slightly longer cycle duration than solution $b$ in the MMA sys- 
tem. In the HIPS system shorter cycle times were associated with better operation of the system. Notice, however, that the optimal solution does have shorter cycle times than the other suboptimal solutions except for solution $b$. The fact that case $b$ operates in a slightly shorter cycle is also reflected in lower inventory costs for this suboptimal solution when compared to the optimal solution. This is explained by the fact that the difference in cycle duration and inventory costs between the two solutions is very small, and it is out weighted by the fact that the optimal solution performs better in terms of transition durations (see Table 13 and inventory costs in Figure 9). If one thing is to be emphasized by this second example in addition to what was found in the HIPS case study, it is the importance of minimizing transition duration, and total transition time to cycle duration ratio.

\section{Sensitivity analysis}

The sensitivity analysis referred to in this section consists in the modification of certain fixed problem parameters. Namely, these parameters are inventory costs, raw material costs, tolerance for actual states values versus desired state values at the end of dynamic transitions as expressed in Eqns. 20 and 21, and hourly demand for grade $A$. The percentage of change in these parameters and the resulting optimal solutions are summarized in Table 14.

Case I. When inventory costs are increased, profit is decreased as expected. The decrease is not proportional to the increase in inventory cost since the amount of polymer produced has to be changed by the optimizer attempting to minimize costs. An over production of grade $D$ when compared against its demand is still present, but it is lower than in the base case. When less polymer is produced the sales term is also affected. The overall effect is seen in the resulting hourly profit. A decrease in cycle duration also occurs, as expected when dealing with higher inventory holding costs. On the other hand, decreased inventory costs cause an expected increase in profit. This is caused by 
cheaper holding costs allowing for longer cycles, more production and therefore more over production of grade $D$. Hence, the increase in profit is caused by the decrease in costs itself and by an increase in hourly sales.

Case II. Raw material costs for monomer and initiator were simultaneously modified. This change has interesting effects. The case where raw material costs were decreased is the only case where a different optimal sequence was obtained. Changing this sequence impacts the transition times. This occurs because lower transition costs allow for longer transition times, while still increasing the hourly profit when compared against the original case. This fact seems to run against what has been found previously in this paper where minimizing transition times was a driver for the optimal solution. This indicates that there is a limit on conditions where all the analysis performed before are valid.

Case III. Modifying the allowed tolerances is equivalent to modifying the duration of transitions. When a tolerance is increased (therefore relaxing the dynamic end point constraints), less time is required for grade transitions, and the opposite is true when tolerances are decreased. Notice that costs were not modified in both cases, and the profit still changes. The $50 \%$ changes correspond to increasing and decreasing the original $2 \%$ tolerance by $1 \%$. Although this change is not large, it is still affects cycle duration and cycle productivity, if productivity is understood as the proportion of the cycle devoted to actually producing the desired polymer grades. The effect seen when tolerances are modified further points out the important role that transitions duration and dynamic behavior of the system play in determining an optimal solution.

Case IV. Changes in the demand for any grade are of particular interest since actual manufacturing operations are always subject to this kind of uncertain behavior. Grade $A$ was chosen because it is the less profitable grade. When the demand is increased, a longer cycle time has to be dedicated to producing this grade (the model used sets grade demand as a lower bound on production). Increasing the portion of the cycle devoted to less profitable grades necessarily decreases profit, since other grades have to be hold during the production of this less profitable product. The optimal sequence is still the 
same which means that product $D$, with the highest production of all is produced before

grade $A$. Because production of grade $A$ takes longer, all $D$ grade has to be hold for a longer amount of time, increasing inventory costs. The optimizer, however, does not change the total cycle duration too much, but actually produces less grade $D$ so less production has to be stored. This negatively affects profit since grade $D$ has the highest selling price. The opposite effect can be observed when the demand of grade $A$ is lowered.

\subsection{Conclusions}

In this work a MINLP formulation was used to simultaneously solve the scheduling and control problems in polymerization reactors during a cyclic manufacturing operation. Process dynamics, in the form of differential equations that were transformed into algebraic equations via a discretization method, were explicitly included in the formulation. Such a formulation was able to calculate the optimal transition duration and profiles, and from this information a transition cost term was computed in the objective function. The dynamic optimization was performed using the SDO method which allows for transitions between unstable operating conditions. Results do not depend on any controller set up since they all correspond to open loop solutions.

Two case studies related to the polymerization industry were included. From these studies it was concluded that the formulation worked for applications close to those expected in real life. From the discussion of those cases it was concluded that transition duration is decisive for the optimal profit achieved, therefore justifying the need to include rigorous dynamics in the simultaneous problem. The superiority of the presented formulation vs. the sequential approach was evident in one of the examples.

A potential extension of this work is the application of the formulation presented to models used to simulate real life operations. Taking the examples in this work as a starting point, the optimization of polymerization operations in non-isothermal mode, and perhaps, using a more representative reactor model will be a next step. Since computational effort invested in solving the case studies was significant, a decomposition approach ex- 
Table 1: Design Information and Scaling Values

\begin{tabular}{lll}
\hline$V$ & 6000 & Reactor volume $[\mathrm{L}]$ \\
$Q_{i}$ & $1.5 \times 10^{3}$ & Initiator flow rate $[\mathrm{L} / \mathrm{s}]$ \\
$C_{m 0}$ & 8.63 & Monomer feed stream concentration $[\mathrm{mol} / \mathrm{s}]$ \\
$C_{b 0}$ & 1.05 & Butadiene feed stream concentration $[\mathrm{L} / \mathrm{s}]$ \\
$C_{I 0}$ & 0.98 & Initiator feed stream concentration $[\mathrm{L} / \mathrm{s}]$ \\
$T$ & 377.5 & Reactor temperature $[\mathrm{K}]$ \\
$k_{d}$ & $7.28 \times 10^{-4}$ & Initiation reaction constant $[1 / \mathrm{s}]$ \\
$k_{I 0}$ & $1.59 \times 10^{-11}$ & Initiation reaction constant $[\mathrm{L} / \mathrm{mol}-\mathrm{s}]$ \\
$k_{I 1}$ & $8.04 \times 10^{2}$ & Initiation reaction constant $[\mathrm{L} / \mathrm{mol}-\mathrm{s}]$ \\
$k_{I 2}$ & $1.61 \times 10^{2}$ & Initiation reaction constant $[\mathrm{L} / \mathrm{mol}-\mathrm{s}]$ \\
$k_{I 3}$ & $8.04 \times 10^{2}$ & Initiation reaction constant $[\mathrm{L} /$ mol-s] \\
$k_{p}$ & $8.04 \times 10^{2}$ & Propagation reaction constant $[\mathrm{L} /$ mol-s $]$ \\
$k_{f s}$ & $2.99 \times 10^{-1}$ & Monomer transfer reaction constant $[\mathrm{L} / \mathrm{mol}-\mathrm{s}]$ \\
$C_{\operatorname{mmax}}$ & 7.31 & Maximum value of monomer concentration $[\mathrm{mol} / \mathrm{l}]$ \\
$C_{I \max }$ & $3 \times 10^{-4}$ & Maximum value of initiator concentration $[\mathrm{mol} / \mathrm{l}]$ \\
$C_{\text {max }}$ & 1.05 & Maximum value of butadiene concentration $[\mathrm{mol} / \mathrm{l}]$ \\
$C_{r \max }$ & $6.29 \times 10^{-11}$ & Maximum value of radical concentration $[\mathrm{mol} / \mathrm{l}]$ \\
$C_{\text {brmax }}$ & $4.97 \times 10^{-12}$ & Maximum value of butadiene radical concentration $[\mathrm{mol} / \mathrm{l}]$ \\
$\mu_{r \max }^{0}$ & $8.66 \times 10^{-8}$ & Maximum value of zero radical death moment \\
$\mu_{b \max }^{0}$ & $4.41 \times 10^{-9}$ & Maximum value of zero butadiene radical death moment \\
$Q_{I \max }$ & $1.5 \times 10^{-3}$ & Maximum value of initiator flow rate $[\mathrm{l} / \mathrm{s}]$ \\
$Q_{\operatorname{mmax}}$ & 1.14 & Maximum value of feed stream flow rate $[\mathrm{l} / \mathrm{s}]$
\end{tabular}

ploiting the nature of the simultaneous problem is desirable. Such an approach might also facilitate the representation of scheduling models beyond the cyclic one presented here.

Table 2: HIPS Grade Design Information. Demand rate is in $[\mathrm{kg} / \mathrm{hr}]$, inventory cost is in $[\$ / \mathrm{kg}-\mathrm{hr}]$, monomer and initiator costs are in $[\$ / \mathrm{lt}$ of feed stream] and prices are in $[\$ / \mathrm{kg}]$

\begin{tabular}{|c|c|c|c|c|c|c|c|}
\hline Grade & $Q[\mathrm{l} / \mathrm{s}]$ & Conv. & Demand $(\mathrm{kg} / \mathrm{hr})$ & Inv. Cost & Monomer Cost & Initiator Cost & Price \\
\hline$A$ & 1.14 & 15 & 50 & 0.15 & 1 & 10 & 3.2 \\
$B$ & 0.75 & 25 & 60 & 0.20 & 1 & 10 & 4.3 \\
$C$ & 0.56 & 35 & 65 & 0.15 & 1 & 10 & 4.5 \\
$D$ & 0.60 & 40 & 70 & 0.10 & 1 & 10 & 5.0 \\
$E$ & 0.53 & 45 & 60 & 0.25 & 1 & 10 & 5.5 \\
\hline
\end{tabular}


Table 3: Simultaneous scheduling and control results for grade transition in a HIPS polymerization CSTR. The optimal cyclic sequence is $E \rightarrow A \rightarrow B \rightarrow C \rightarrow D$. The objective function value is $\$ 1456 / \mathrm{hr}$ and $32.3 \mathrm{~h}$ of total cycle time.

\begin{tabular}{|c|c|c|c|c|c|}
\hline Product & Process T $[\mathrm{h}]$ & production $[\mathrm{kg}]$ & Trans $\mathrm{T}[\mathrm{h}]$ & $\mathrm{T}$ start $[\mathrm{h}]$ & $\mathrm{T}$ end $[\mathrm{h}]$ \\
\hline$E$ & 2.48 & 1937 & 1.34 & 0 & 3.83 \\
$A$ & 2.87 & 1614 & 1.15 & 3.83 & 7.85 \\
$B$ & 3.17 & 1937 & 1.11 & 7.85 & 12.14 \\
$C$ & 3.10 & 2099 & 0.58 & 12.14 & 15.82 \\
$D$ & 15.81 & 11370 & 0.67 & 15.82 & 32.29 \\
\hline
\end{tabular}

Table 4: Performance indicators for HIPS CSTR optimal and other suboptimal feasible solutions

\begin{tabular}{|c|c|c|c|c|c|c|c|}
\hline Solution & sequence & Profit & Tc $[\mathrm{h}]$ & $\frac{\text { Transtime }}{\text { CycTime }}$ & $\frac{w_{D}}{w_{a} \text { all }}$ & $\frac{w_{A, B, C, E}}{\text { demand }}$ & $\frac{w_{D}}{\text { demand }}$ \\
\hline Optimal & $E \rightarrow A \rightarrow B \rightarrow C \rightarrow D$ & 1456 & 32.3 & 0.15 & 0.60 & 1.0 & 5.0 \\
Sol.B & $D \rightarrow A \rightarrow B \rightarrow C \rightarrow E$ & 1352 & 33.0 & 0.16 & 0.59 & 1.0 & 4.9 \\
Sol.C & $E \rightarrow B \rightarrow A \rightarrow C \rightarrow D$ & 1221 & 36.2 & 0.17 & 0.59 & 1.0 & 4.8 \\
Sol.D & $A \rightarrow B \rightarrow C \rightarrow D \rightarrow E$ & 1155 & 37.2 & 0.18 & 0.59 & 1.0 & 4.7 \\
Sol.E & $E \rightarrow A \rightarrow C \rightarrow B \rightarrow D$ & 1101 & 38.1 & 0.19 & 0.58 & 1.0 & 4.7 \\
Sol.F & $B \rightarrow A \rightarrow E \rightarrow C \rightarrow D$ & 1045 & 39.0 & 0.20 & 0.58 & 1.0 & 4.6 \\
\hline
\end{tabular}

Table 5: Dominant eigenvalues for linearized steady states corresponding to different grade operating conditions for the base case $(V=6000 \mathrm{~L})$ and the modified case $(V=2500 \mathrm{~L})$.

\begin{tabular}{|c|c|c|}
\hline Grade & $\begin{array}{c}\text { Dominant Eigenvalue } \\
\text { (Base case) }\end{array}$ & $\begin{array}{c}\text { Dominant Eigenvalue } \\
\text { (Modified case) }\end{array}$ \\
\hline$A$ & $-1.59 \times 10^{-4}$ & $-3.92 \times 10^{-4}$ \\
$B$ & $-1.02 \times 10^{-4}$ & $-2.55 \times 10^{-4}$ \\
$C$ & $-7.97 \times 10^{-5}$ & $-2.10 \times 10^{-4}$ \\
$D$ & $-7.30 \times 10^{-5}$ & $-1.88 \times 10^{-4}$ \\
$E$ & $-6.96 \times 10^{-5}$ & $-1.78 \times 10^{-4}$ \\
\hline
\end{tabular}

Table 6: Sequential scheduling and control results for grade transition in a HIPS polymerization CSTR. The objective function value is $\$ 1416$ and $33 \mathrm{~h}$ of total cycle time.

\begin{tabular}{|c|c|c|c|c|c|}
\hline Product & Process T $[\mathrm{h}]$ & production $[\mathrm{kg}]$ & Trans T $[\mathrm{h}]$ & $\mathrm{T}$ start $[\mathrm{h}]$ & $\mathrm{T}$ end $[\mathrm{h}]$ \\
\hline$C$ & 3.16 & 2141 & 0.58 & 0 & 3.75 \\
$D$ & 16.03 & 11530 & 0.67 & 3.75 & 20.44 \\
$E$ & 2.54 & 1977 & 1.54 & 20.44 & 24.52 \\
$A$ & 2.93 & 1647 & 1.14 & 24.52 & 28.60 \\
$B$ & 3.23 & 1977 & 1.11 & 28.60 & 32.95 \\
\hline
\end{tabular}


Table 7: Comparison between simultaneous and sequential solutions

\begin{tabular}{|c|c|c|c|c|}
\hline Method & Sales $[\$ / \mathrm{hr}]$ & inv. costs $[\$ / \mathrm{hr}]$ & trans. costs $[\$ / \mathrm{hr}]$ & Profit $[\$ / \mathrm{hr}]$ \\
\hline Simultaneous & 2634.70 & 941.00 & 404.68 & 1455.55 \\
Sequential & 2801.24 & 959.99 & 414.19 & 1416.33 \\
\hline
\end{tabular}

Table 8: Comparison between simultaneous and modified sequential solutions

\begin{tabular}{|c|c|c|c|c|}
\hline Method & Sales $[\$ / \mathrm{hr}]$ & inv. costs $[\$ / \mathrm{hr}]$ & trans. costs $[\$ / \mathrm{hr}]$ & Profit $[\$ / \mathrm{hr}]$ \\
\hline Simultaneous & 2634.70 & 941.00 & 404.68 & 1455.55 \\
Sequential & 2801.38 & 940.79 & 405.17 & 1454.86 \\
\hline
\end{tabular}

Table 9: Simultaneous scheduling and control results for grade transition in a HIPS polymerization CSTR. The objective function value is $\$ 906 / \mathrm{hr}$ and $40 \mathrm{~h}$ of total cycle time.

\begin{tabular}{|c|c|c|c|c|c|}
\hline Product & Process T $[\mathrm{h}]$ & production $[\mathrm{kg}]$ & Trans T $[\mathrm{h}]$ & T start $[\mathrm{h}]$ & T end $[\mathrm{h}]$ \\
\hline$A$ & 3.57 & 2008 & 3 & 0 & 6.57 \\
$D$ & 10.67 & 7686 & 3 & 6.57 & 20.26 \\
$C$ & 3.86 & 2610 & 3 & 20.26 & 27.11 \\
$E$ & 3.10 & 2409 & 3 & 27.11 & 33.21 \\
$B$ & 3.92 & 2409 & 3 & 33.21 & 40.15 \\
\hline
\end{tabular}

Table 10: Steady-state design and scaling information.

\begin{tabular}{lll}
\hline$Q_{m}$ & 1 & Monomer feed stream $\left[\mathrm{m}^{3} / \mathrm{h}\right]$ \\
$\mathrm{V}$ & 0.1 & Reactor volume $\left[\mathrm{m}^{3}\right]$ \\
$C_{i_{i n}}$ & 8 & Feed stream initiator concentration $\left[\mathrm{kmol} / \mathrm{m}^{3}\right]$ \\
$M_{m}$ & 100.12 & Monomer molecular weight $[\mathrm{kg} / \mathrm{kmol}]$ \\
$C_{m_{i n}}$ & 6 & Feed stream monomer concentration $\left[\mathrm{kmol} / \mathrm{m}^{3}\right]$ \\
$f^{*}$ & 0.58 & Initiator efficiency \\
$k_{t c}$ & $1.3281 \times 10^{10}$ & Termination by coupling rate constant $\left[\mathrm{m}^{3} /(\mathrm{kmol}-\mathrm{h})\right]$ \\
$k_{t d}$ & $1.093 \times 10^{11}$ & Termination by disproportion rate constant $\left[\mathrm{m}^{3} /(\mathrm{kmol}-\mathrm{h})\right]$ \\
$k_{i}$ & $1.0255 \times 10^{-1}$ & Initiation rate constant $[1 / \mathrm{h}]$ \\
$k_{p}$ & $2.4952 \times 10^{6}$ & Propagation rate constant $\left[\mathrm{m}^{3} /(\mathrm{kmol}-\mathrm{h})\right]$ \\
$k_{f m}$ & $2.4522 \times 10^{3}$ & Chain transfer to monomer rate constant $\left[\mathrm{m}^{3} /(\mathrm{kmol}-\mathrm{h})\right]$ \\
$\hat{C}_{m}$ & 5.7768 & Maximum value of monomer concentration $\left[\mathrm{kmol} / \mathrm{m}^{3}\right]$ \\
$\hat{C}_{i}$ & 0.41534 & Maximum value of initiator concentration $\left[\mathrm{kmol} / \mathrm{m}^{3}\right]$ \\
$\hat{D}_{0}$ & $5.4794 \times 10^{-3}$ & Maximum value of zeroth moment \\
$\hat{D}_{1}$ & 82.219 & Maximum value of first moment \\
$\hat{Q}_{i}$ & 0.05245 & Maximum value of initiator flow rate $\left[\mathrm{m}^{3} / \mathrm{h}\right]$ \\
$\hat{Q}_{m}$ & 1 & Maximum value of monomer flow rate $\left[\mathrm{m}^{3} / \mathrm{h}\right]$ \\
\hline
\end{tabular}


Table 11: Grade design information. The demand rate is in $[\mathrm{kg} / \mathrm{h}]$ the price in $[\$ / \mathrm{kg}]$, the inventory cost in $[\$ / \mathrm{kg}-\mathrm{h}]$ and the raw material costs are in $\left[\$ / \mathrm{m}^{3}\right.$ of feedstream].

\begin{tabular}{cccccccc}
\hline Grade & $Q_{i}\left[\mathrm{~m}^{3} / \mathrm{hr}\right]$ & MWD & Demand $(\mathrm{kg} / \mathrm{hr})$ & Inv. Cost & Mono. Cost & Init. Cost & Price \\
\hline$A$ & 0.05245 & 15000 & 0.8 & 1 & 10 & 500 & 100 \\
$B$ & 0.01673 & 25000 & 0.7 & 1.2 & 10 & 500 & 120 \\
$C$ & 0.006863 & 35000 & 1 & 1.2 & 10 & 500 & 130 \\
$D$ & 0.003114 & 45000 & 0.8 & 1.5 & 10 & 500 & 150 \\
\hline
\end{tabular}

Table 12: Simultaneous scheduling and control results for grade transition in a MMA polymerization CSTR. The objective function value is $\$ 66 / \mathrm{hr}$ and $172 \mathrm{~h}$ of total cycle time.

\begin{tabular}{|c|c|c|c|c|c|}
\hline Product & Process T $[\mathrm{h}]$ & production $[\mathrm{kg}]$ & Trans T $[\mathrm{h}]$ & T start $[\mathrm{h}]$ & T end $[\mathrm{h}]$ \\
\hline$D$ & 56.46 & 282.30 & 5.73 & 0 & 62.19 \\
$A$ & 34.43 & 137.73 & 4.62 & 62.19 & 101.24 \\
$B$ & 24.10 & 120.51 & 2.23 & 101.24 & 127.58 \\
$C$ & 43.04 & 172.16 & 1.56 & 127.57 & 172.16 \\
\hline
\end{tabular}

Table 13: Performance indicators for MMA optimal and other feasible solutions

\begin{tabular}{|c|c|c|c|c|c|c|c|}
\hline Solution & sequence & Profit & Tc $[\mathrm{h}]$ & $\frac{\text { Transtime }}{\text { CycTime }}$ & $\frac{w_{D}}{w_{a} l l}$ & $\frac{w_{A, B, C}}{\text { demand }}$ & $\frac{w_{D}}{\text { demand }}$ \\
\hline Optimal & $D \rightarrow A \rightarrow B \rightarrow C$ & 66.0 & 172.2 & 0.08 & 0.40 & 1.0 & 2.05 \\
Sol.b & $D \rightarrow C \rightarrow B \rightarrow A$ & 65.6 & 160.0 & 0.09 & 0.39 & 1.0 & 1.99 \\
Sol.c & $A \rightarrow B \rightarrow C \rightarrow D$ & 65.1 & 160.0 & 0.09 & 0.39 & 1.0 & 1.98 \\
Sol.d & $D \rightarrow B \rightarrow A \rightarrow C$ & 64.8 & 160.0 & 0.09 & 0.39 & 1.0 & 1.97 \\
Sol.e & $D \rightarrow A \rightarrow C \rightarrow B$ & 63.2 & 160.0 & 0.10 & 0.38 & 1.0 & 1.91 \\
\hline
\end{tabular}

Table 14: Changes in values of fixed parameters and their effects on the optimal solution for the MMA case study. $x_{t o l}$ is the product quality band width.

\begin{tabular}{|c|l|c|c|c|c|}
\hline Case & Parameter change & Opt. Sequence & Profit $[\$ / \mathrm{hr}]$ & Cyc. time [hrs] & Trans. time [hrs] \\
\hline Base & None & $D \rightarrow A \rightarrow B \rightarrow C$ & 66.0 & 172.2 & 14.13 \\
Ia & $+50 \%$ Inv. Cost. & $D \rightarrow A \rightarrow B \rightarrow C$ & 60.0 & 140.1 & 14.13 \\
Ib & $-50 \%$ Inv. Cost. & $D \rightarrow A \rightarrow B \rightarrow C$ & 85.9 & 244.3 & 14.13 \\
IIa & $+20 \%$ Raw M. Cost. & $D \rightarrow A \rightarrow B \rightarrow C$ & 61.9 & 182.3 & 14.13 \\
IIb & $-20 \%$ Raw M. Cost. & $B \rightarrow A \rightarrow C \rightarrow D$ & 70.4 & 161.4 & 14.63 \\
IIIa & $+50 \% x_{t o l}$ & $D \rightarrow A \rightarrow B \rightarrow C$ & 62.6 & 180.7 & 15.16 \\
IIIb & $-50 \% x_{t o l}$ & $D \rightarrow A \rightarrow B \rightarrow C$ & 68.1 & 170.0 & 13.46 \\
IVa & $+20 \%$ Demand $A$ & $D \rightarrow A \rightarrow B \rightarrow C$ & 62.4 & 171.7 & 14.13 \\
IVb & $-20 \%$ Demand $A$ & $D \rightarrow A \rightarrow B \rightarrow C$ & 69.9 & 173.5 & 14.13 \\
\hline
\end{tabular}




\section{Transition period}

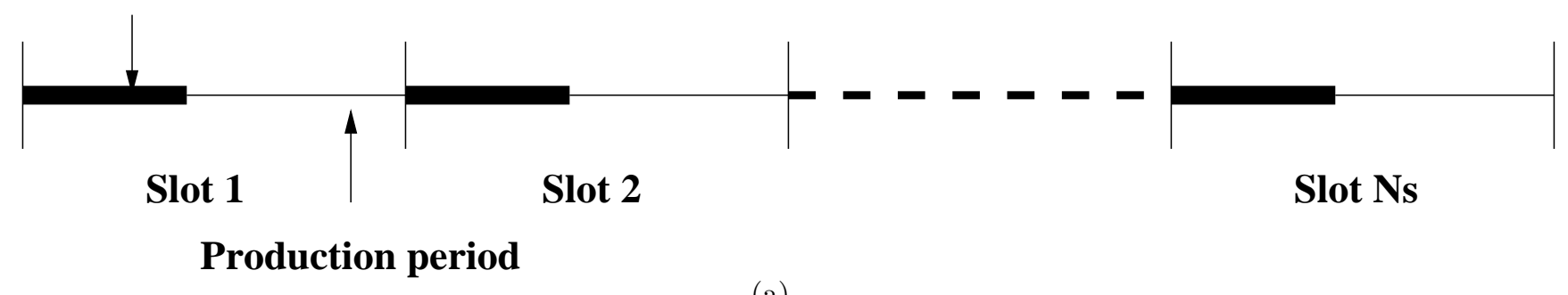

(a)

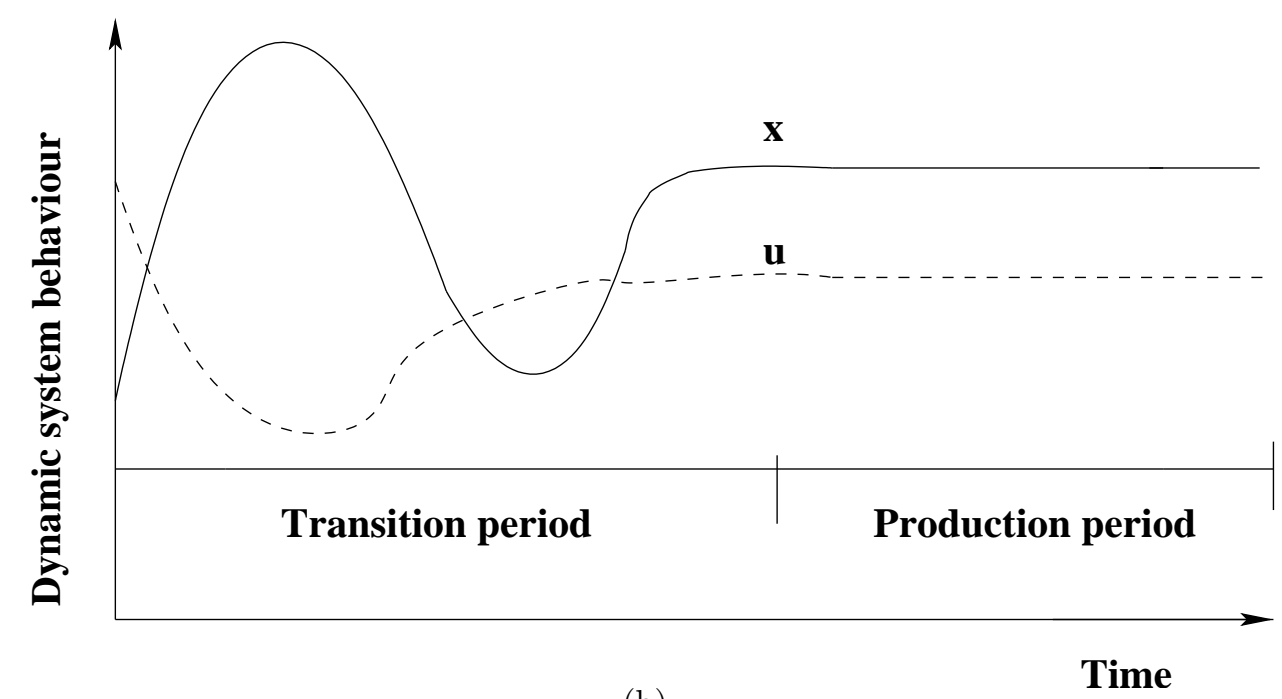

(b)

Figure 1: (a) The cyclic time is divided into slots and within each slot a steady-state production period is followed by a transition period. (b) Within each slot the system states $x$ and the manipulated variables $u$ remain constant. However during the transition period the manipulated variables change and so does the system states. 


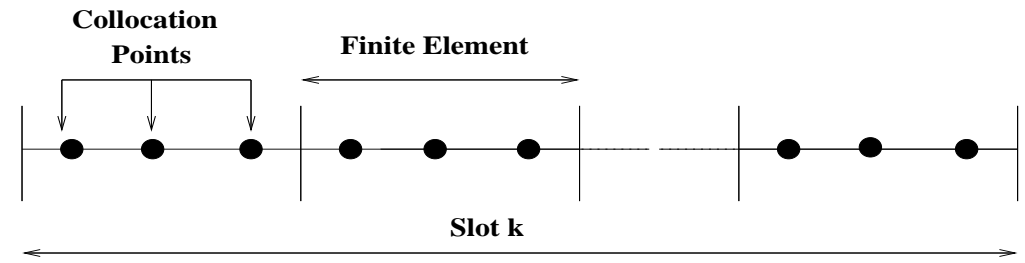

Figure 2: Simultaneous discretization approach for dealing with dynamic optimization problems. Each slot $k$ is divided into $N_{f e}$ finite elements. Within each finite element $f$ a set of $N_{c p}$ collocation points $c$ is selected. 


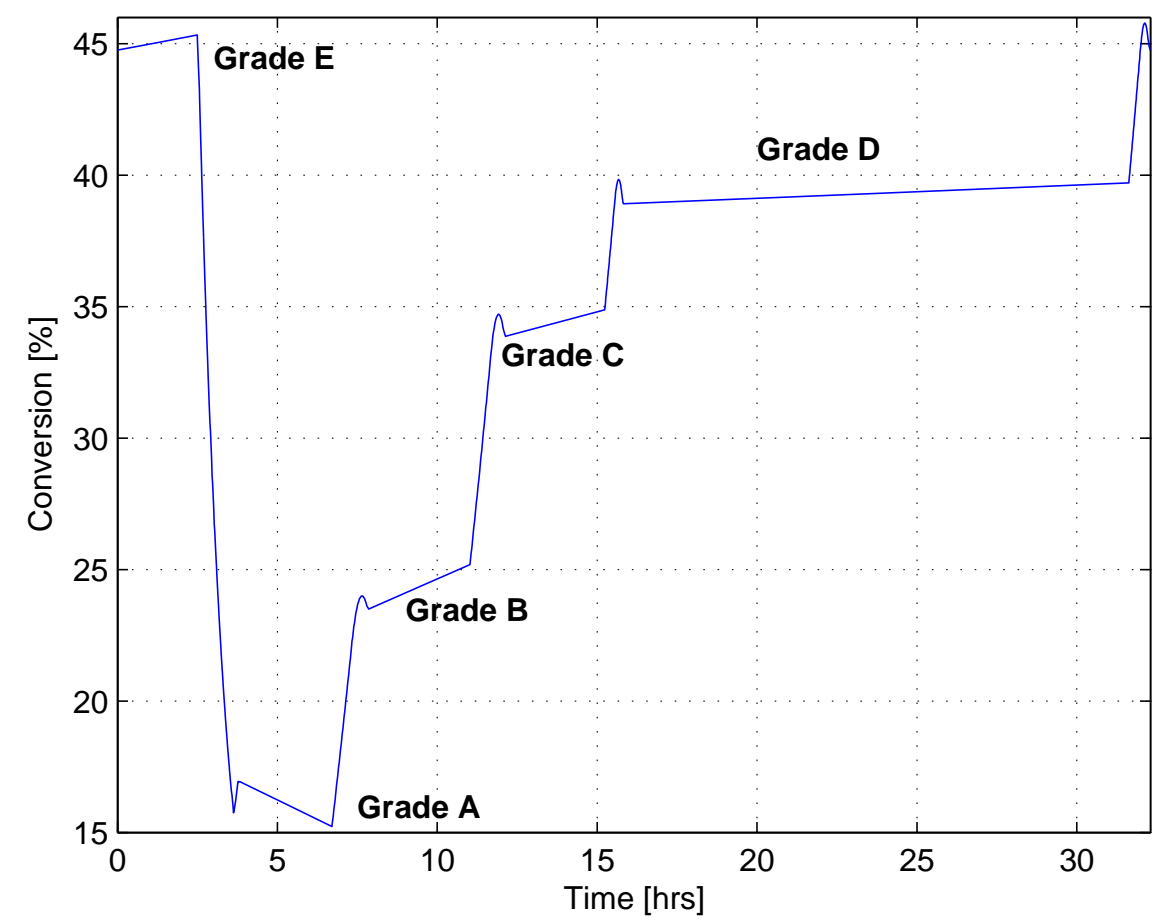

(a)

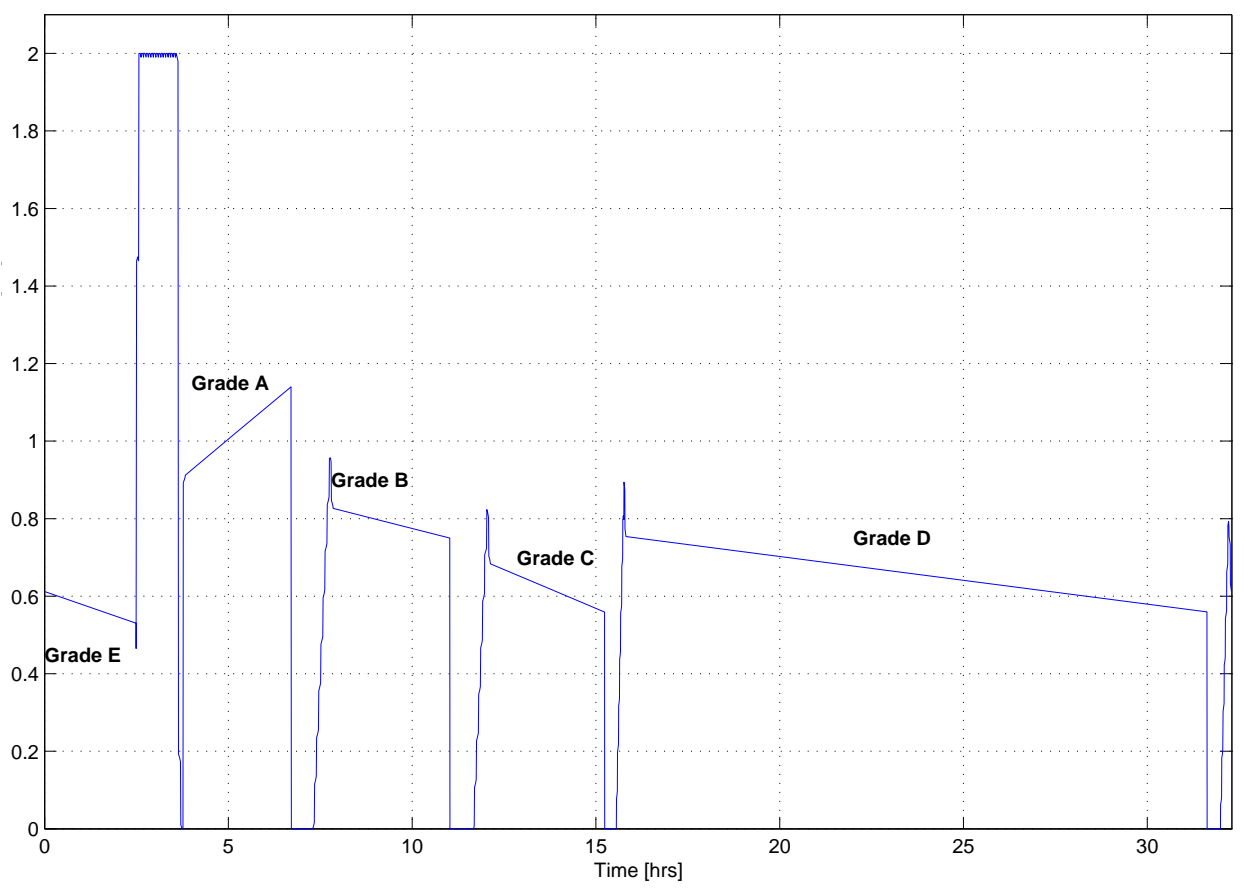

(b)

Figure 3: Monomer conversion (a) and feed stream flow rate $(\mathrm{Qm})$ (b) profiles during production cycle. Note: Conversion during production stages in figure (a) is kept within upper and lower quality bounds 


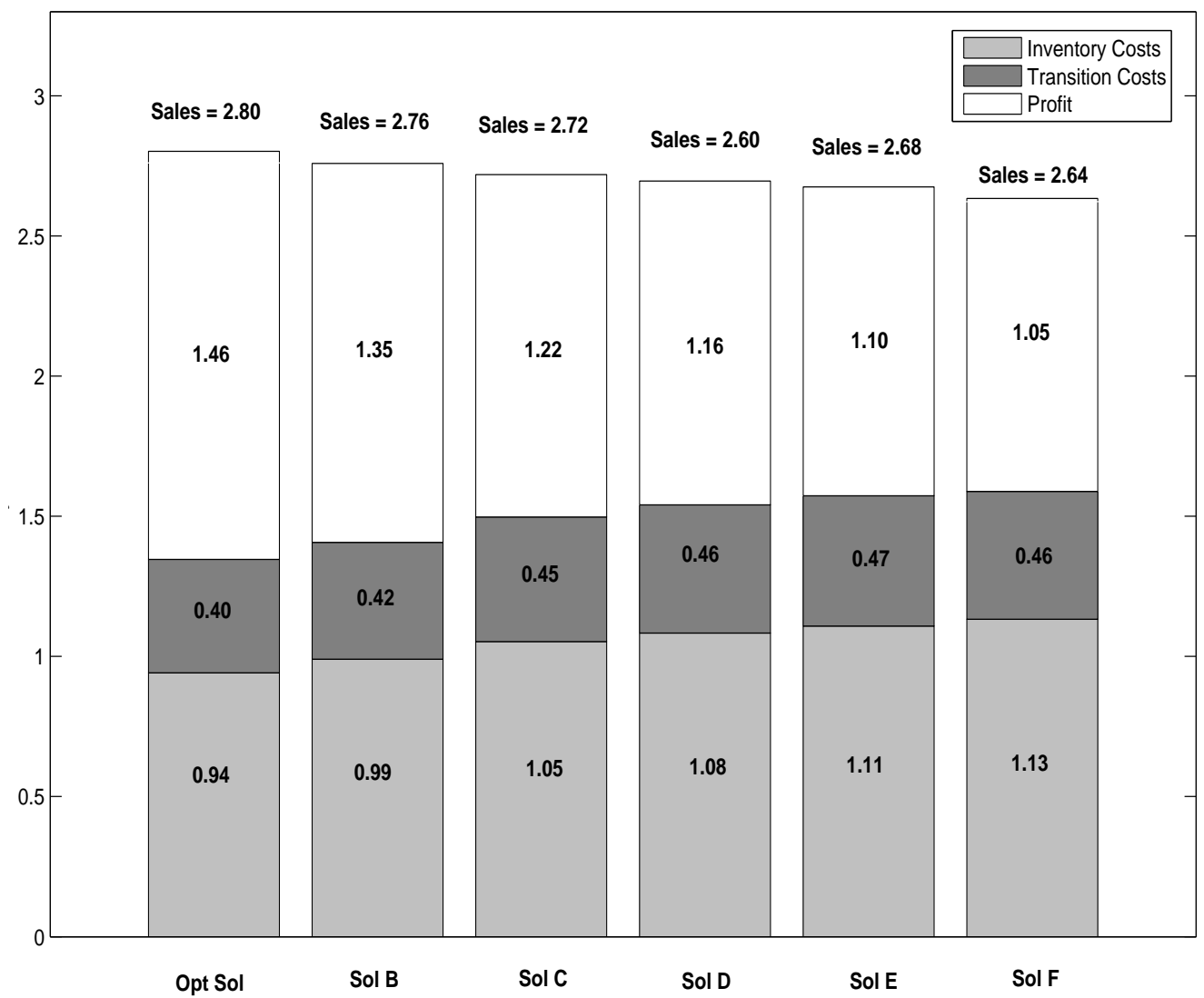

Figure 4: Costs and Profit balance of different solutions for the HIPS case study 


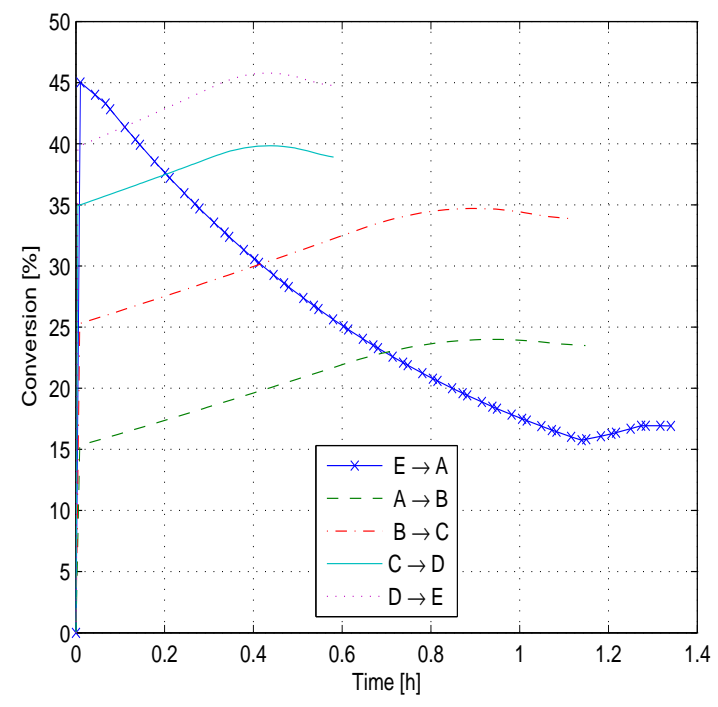

(a)

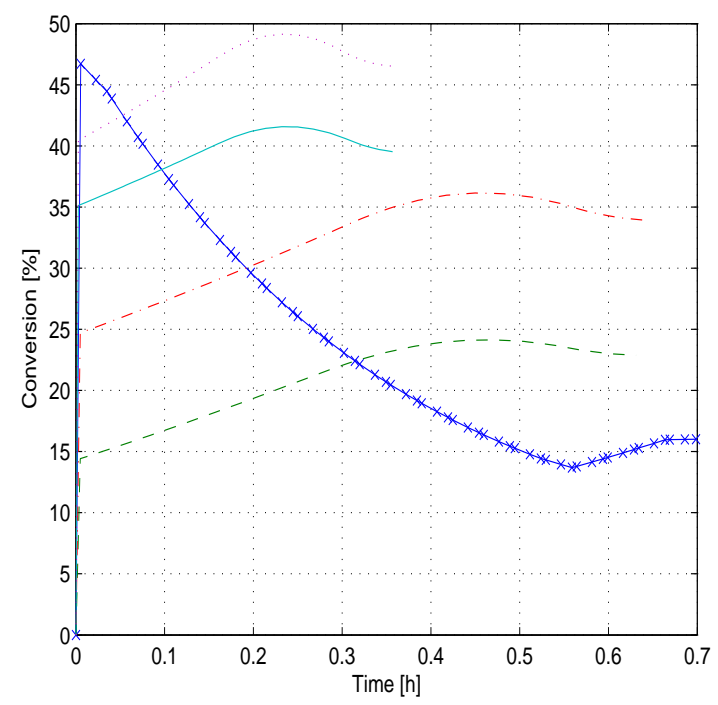

(c)

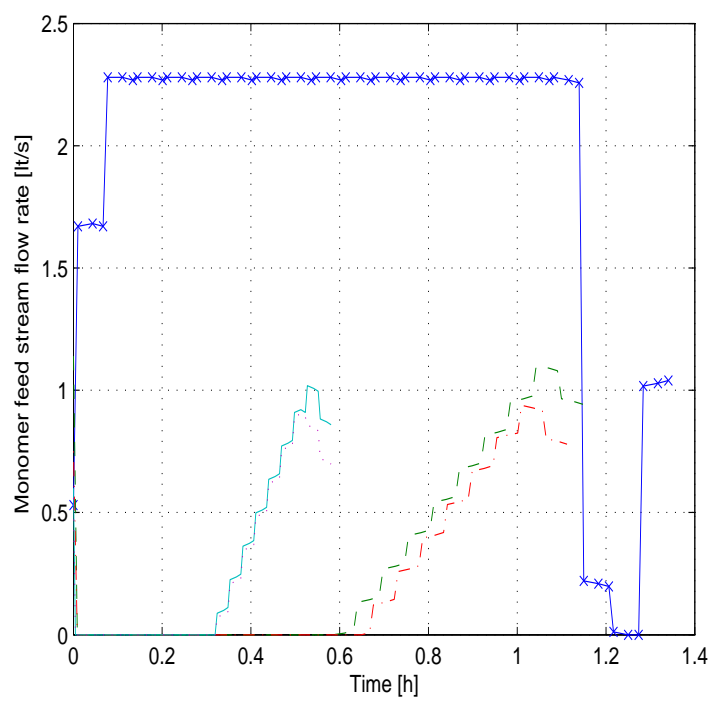

(b)

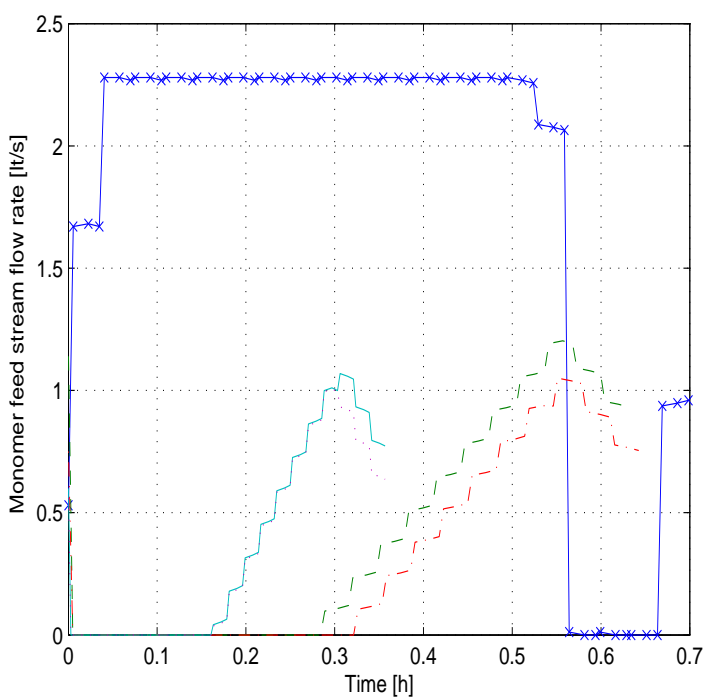

(d)

Figure 5: Conversion (a) and monomer feed stream flow rate (b) profiles of chosen grade transition in original (reactor volume $=6000 \mathrm{lts}$ ). Conversion (c) and monomer feed stream flow rate $(\mathrm{d})$ profiles of chosen grade transition in modified systems (reactor volume $=2500$ lts). 


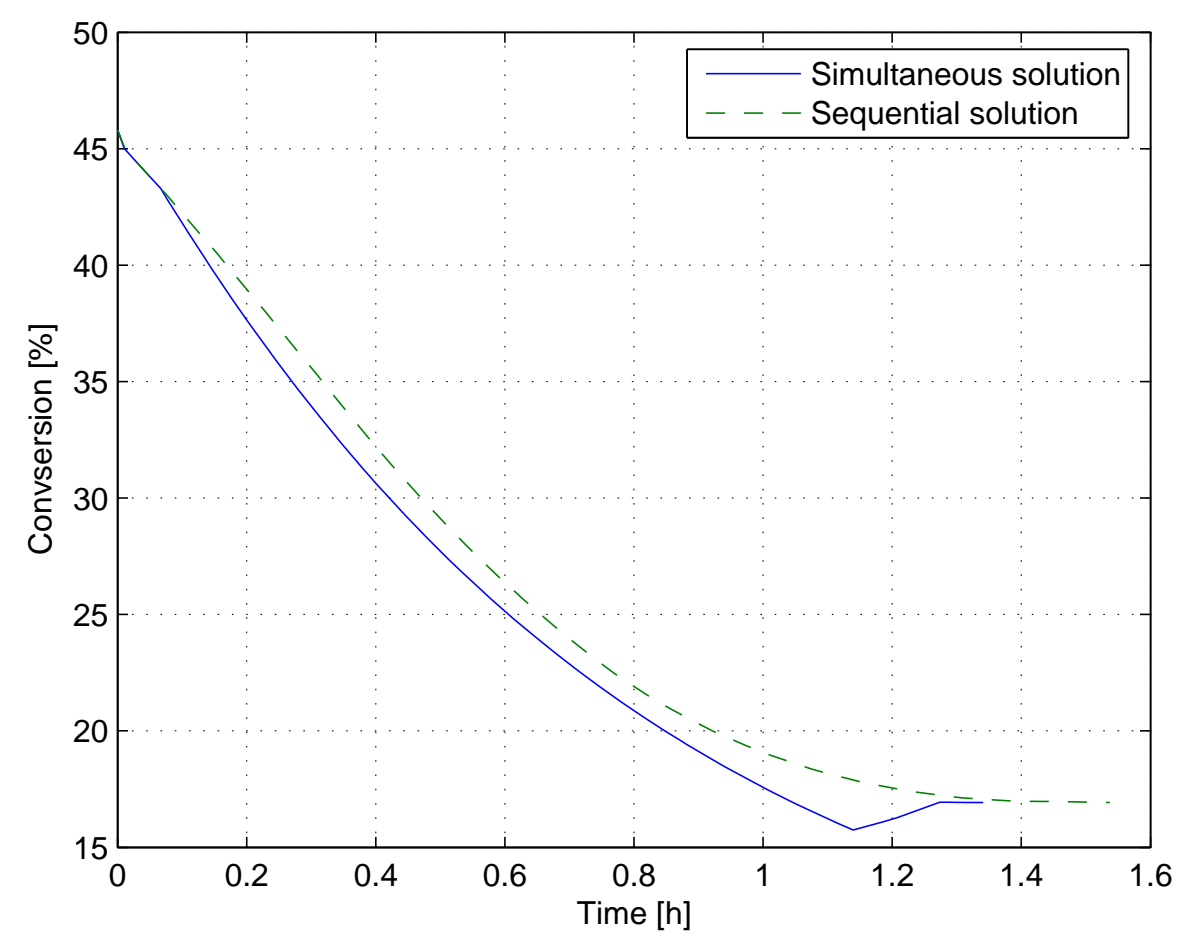

(a)

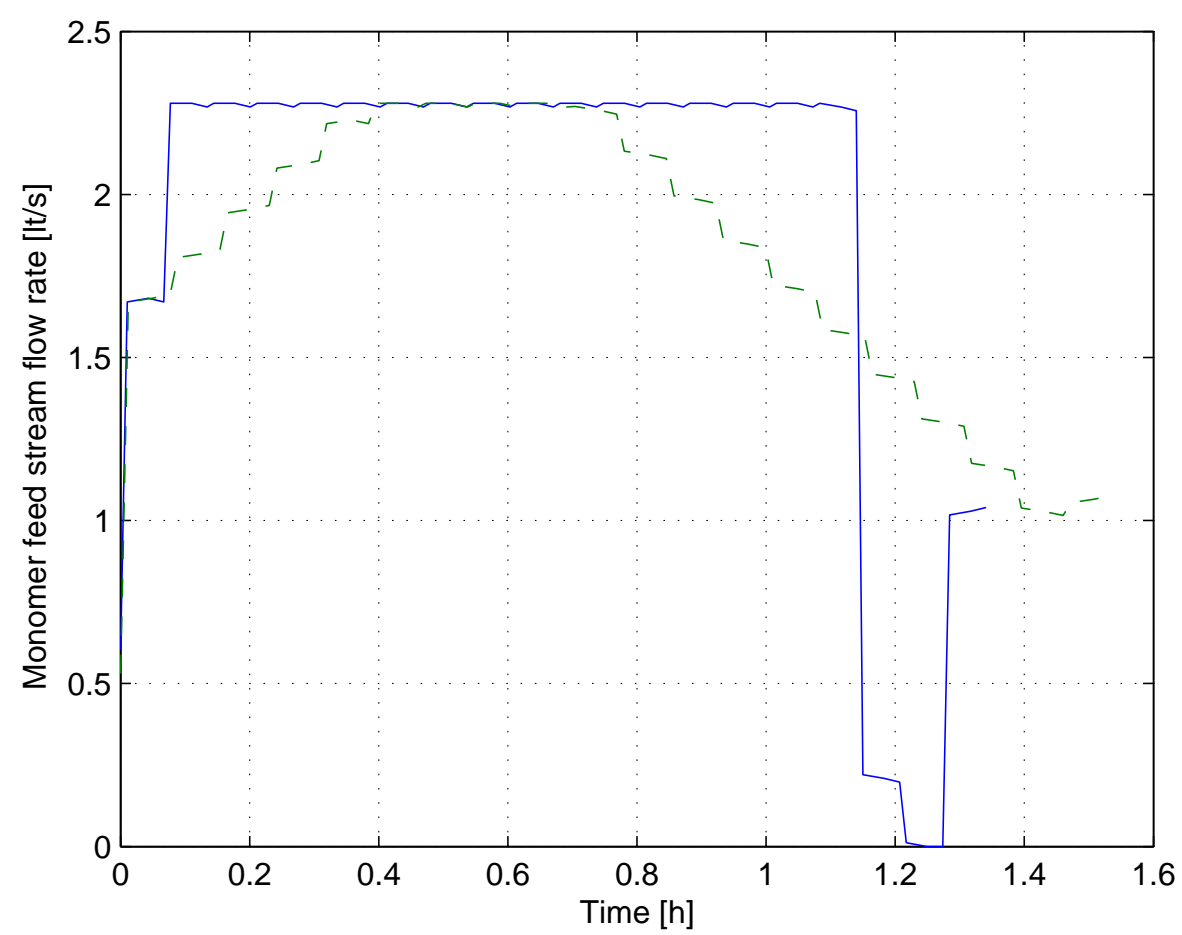

(b)

Figure 6: Conversion (a) and Monomer feed stream flow rate (b) profiles of slot 1 transition for simultaneous and sequential solutions 


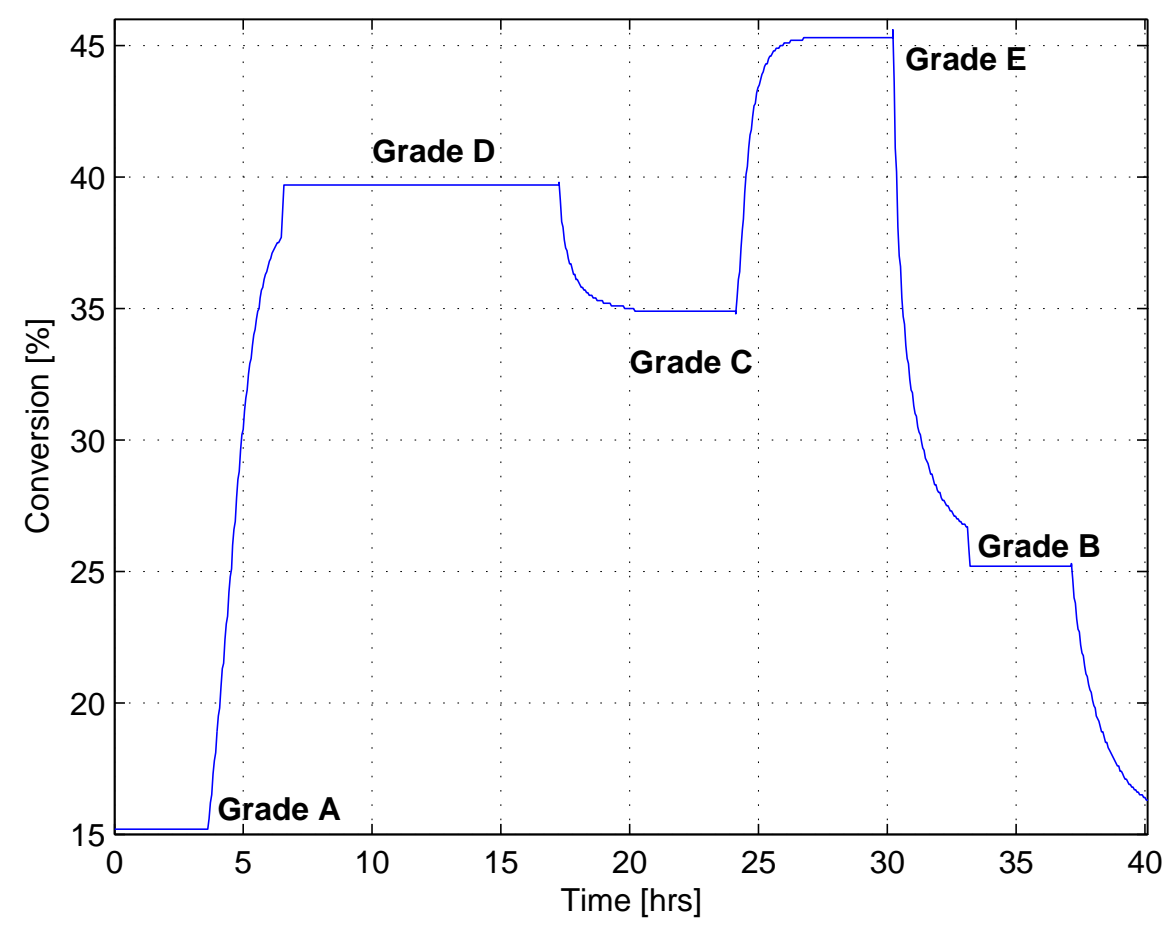

(a)

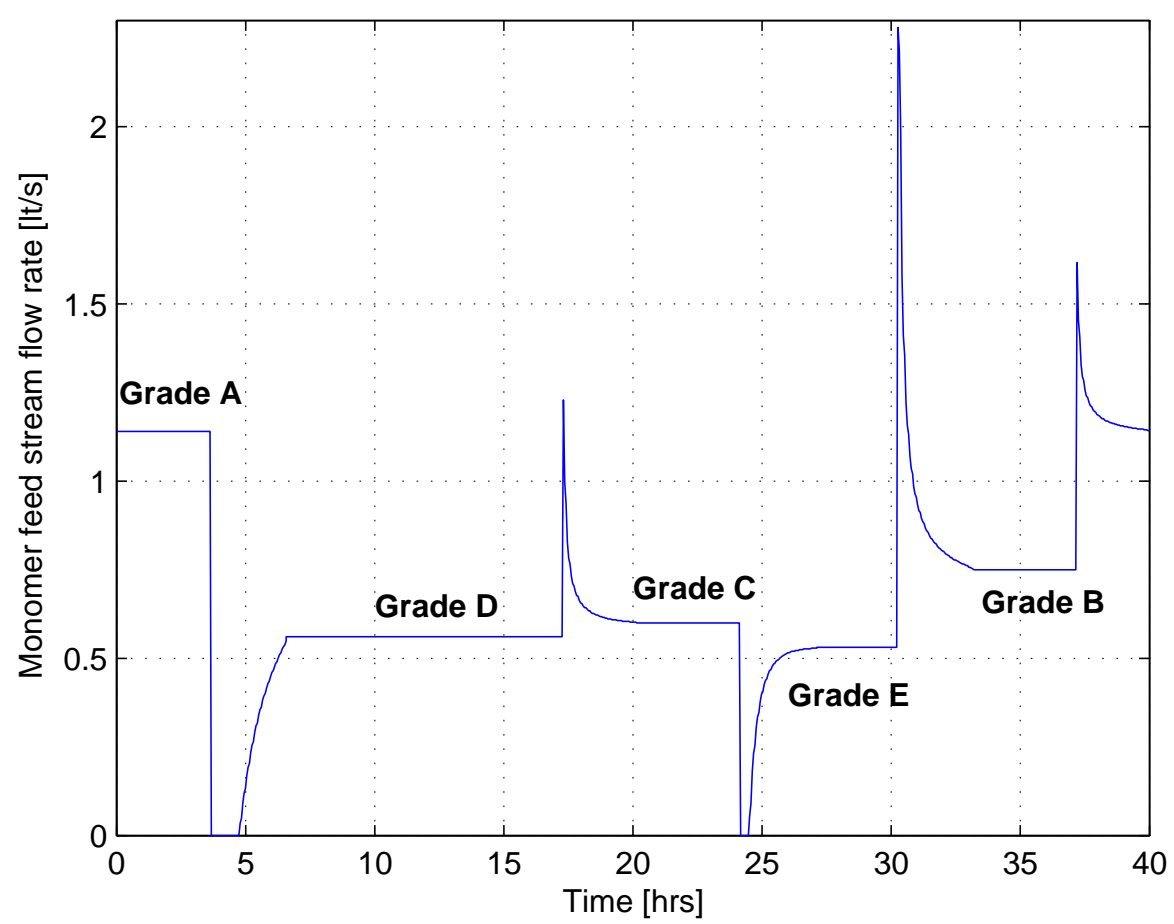

(b)

Figure 7: Monomer conversion (a) and feed stream flow rate $\left(Q_{m}\right)$ (b) profiles during production cycle for iterative solution. 


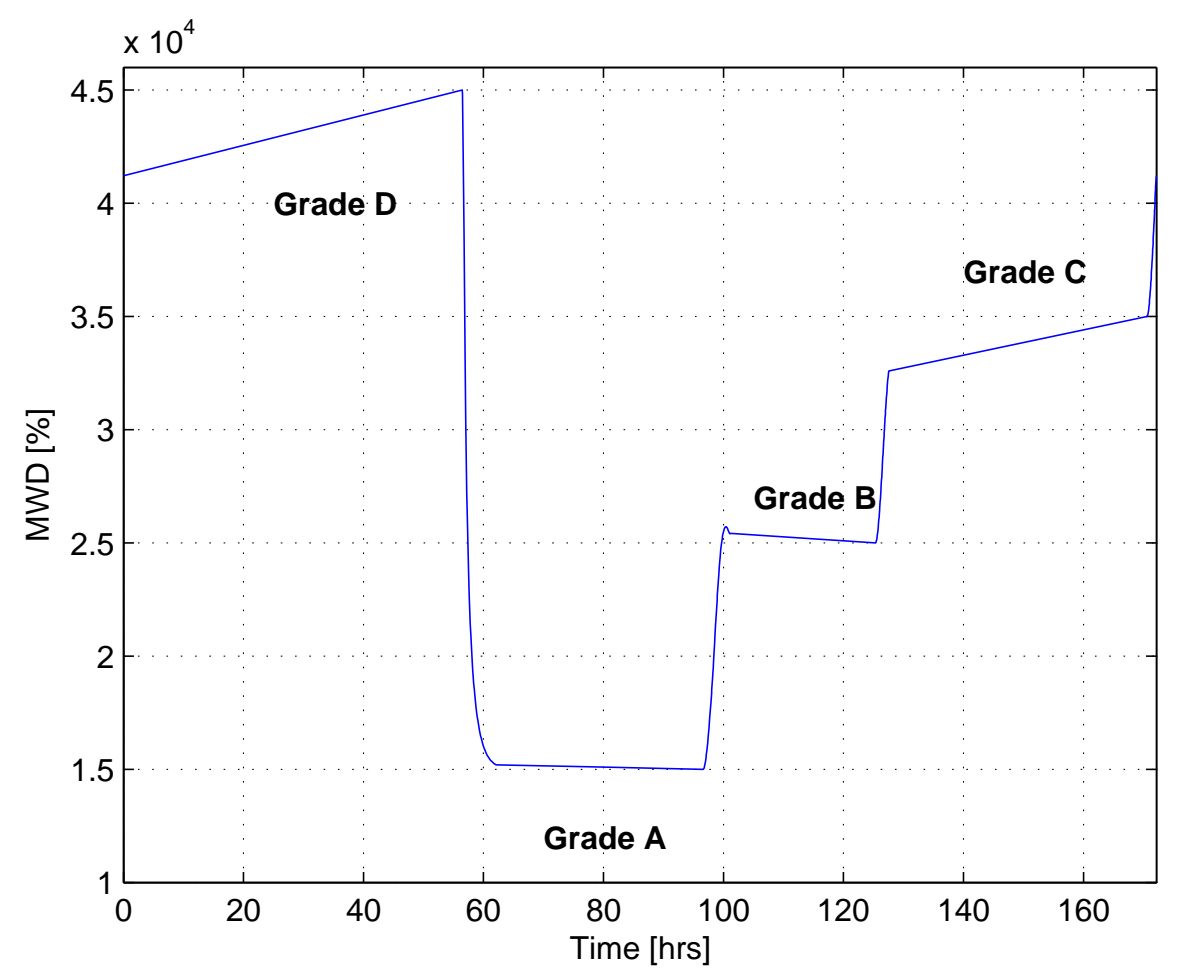

(a)

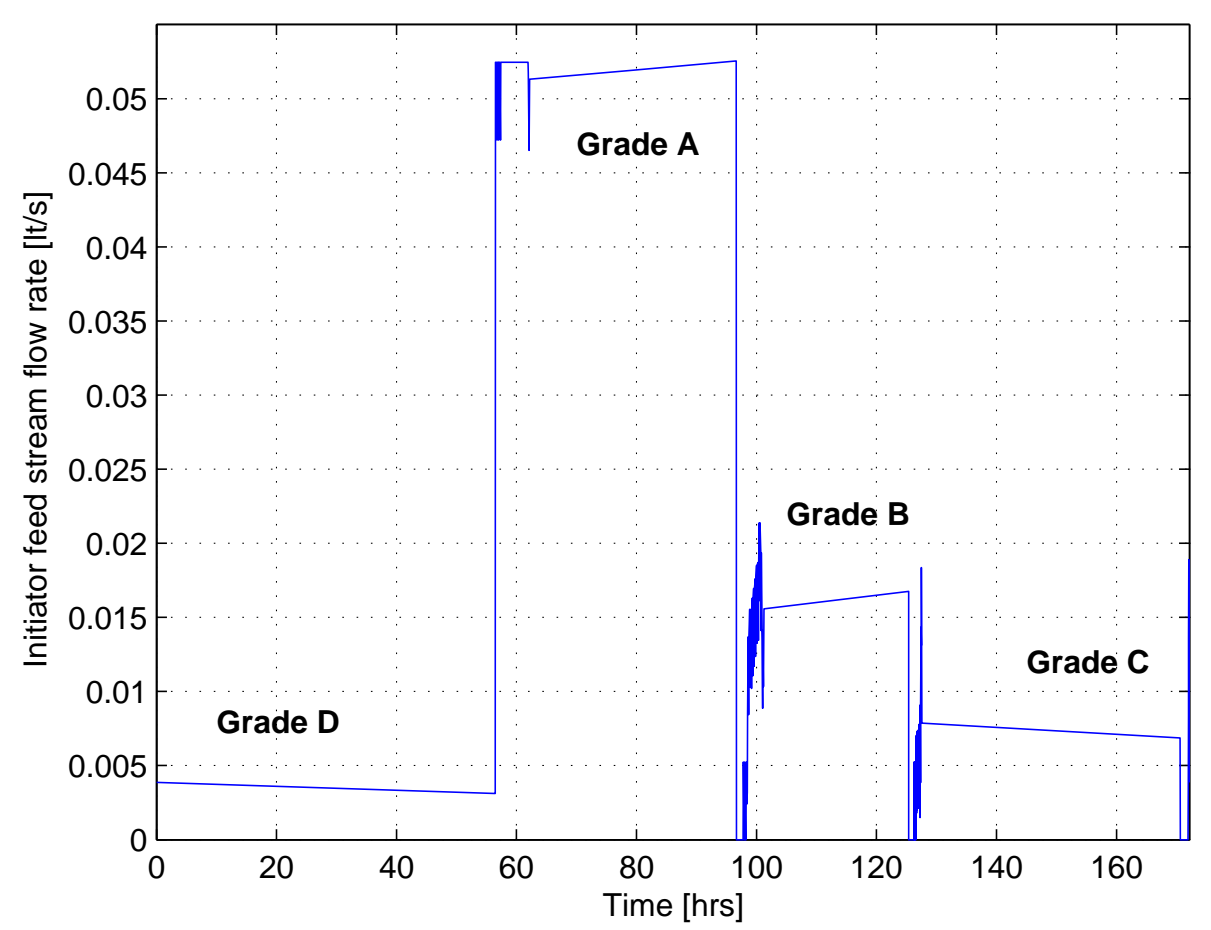

(b)

Figure 8: Molecular weight distribution (a) and feed stream flow rate $\left({ }_{Q} m\right)$ (b) profiles during production cycle. Note: MWD during production stages in figure (a) is kept within upper and lower quality bounds 


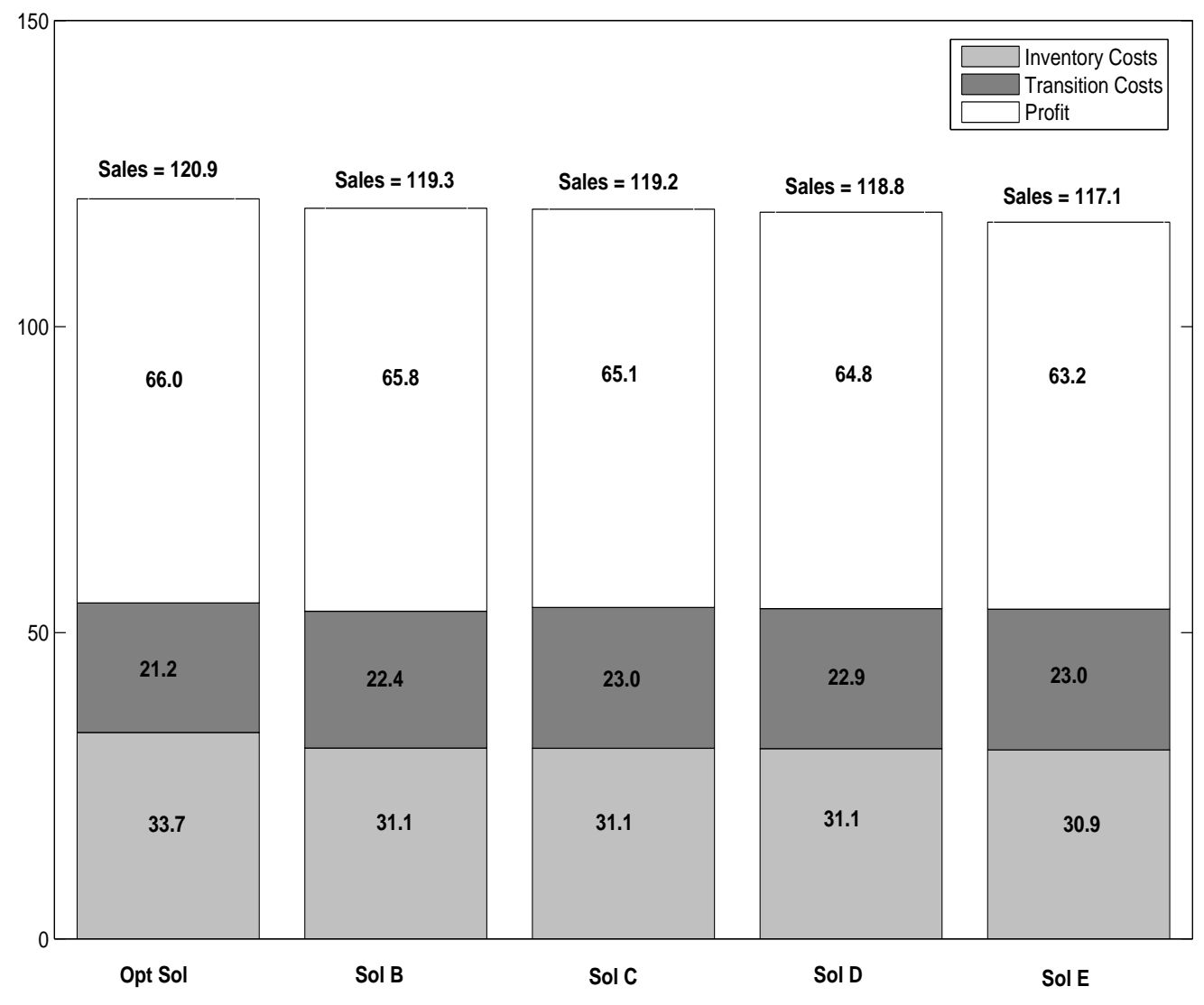

Figure 9: Costs and Profit balance of different solutions for the MMA case study 


\section{References}

[1] R. Mahadevan, F.J. Doyle III, A.C. Allcock. Control-Relevant Scheduling of Polymer Grade Transitions.AIChE Journal, 48(8):1754-1764, 2002.

[2] D. Feather, D. Harrell, R. Liberman, and F.J. Doyle. Hybrid Approach to Polymer Grade Transition Control. AIChE J., 50:(10):2502-2513,2004

[3] R.H. Nystrom, R. Franke, I.Harjunkiski, and A.Kroll. Production Campaign Planning including Grade Transition Sequencing and Dynamic Optimization.Comput.Chem.Eng.,29(10):2163-2179,2005.

[4] B.V. Mishra, E. Mayer, J. Raisch, A. Kienle. Short-Term Scheduling of Batch Processes. A Comparative Study of Different Approaches. Ind. Eng. Chem. Res., 44:40224034, 2005.

[5] A. Flores-Tlacuahuac, L.T. Biegler, E. Saldívar Guerra. Optimal Grade Transitions in the HIPS Polymerization Process.Pendiente.

[6] A. Flores-Tlacuahuac, V. Zavala-Tejeda, E. Saldívar Guerra. Complex Nonlinear Behavior in the Full-Scale High-Impact Polyestyrene Process. Ind. Eng. Chem. Res., 44:2803-2814, 2005.

[7] R.J. Allgor, P.I. Barton. Mixed-integer dynamic optimization: problem formulation.Comput.Chem.Eng.,23(4-5):567-584,1999.

[8] A. Prata, J.Oldenburg, W. Marquardt, R. Nystrom, A. Kroll. Integrated Scheduling and Dynamic Optimization of Grade Transitions for a Continuous Polymerization Reactor. Submitted for Publication 2006.

[9] A. Flores-Tlacuahuac, I.E. Grossmann. Simultaneous Cyclic Scheduling and Control of a Multiproduct CSTR. Submitted for publication 2006.

[10] L.T. Biegler. Optimization strategies for complex process models.Advances in Chemical Engineering, 18:197-256,1992. 
[11] M.A. Duran and I.E. Grossmann. An outer approximation algorithm for a class of mixed integer nonlinear programs. Math.Prog., 36:307,1986.

[12] J. Viswanathan and I.E. Grossmann. A Combined Penalty Function and Outer Approximation Method for MINLP Optimization. Comput.Chem.Eng.,14(7):769782,1990 .

[13] B.Finlayson. Nonlinear Analysis in Chemical Engineering. McGraw-Hill, New York, 1980.

[14] J. Villadsen and M.Michelsen. Solution of Differential Equation Models by Polynomial Approximation. Prentice-Hall,1978.

[15] A. Flores-Tlacuahuac, J.C. Verazaluce-García, E. Saldívar-Guerra. Steady-State Nonlinear Bifurcation Analysis of a High Impact Polystyrene Continuous Stirred Reactor.Ind.Che.Eng.Res.,39:1972-1979,2000.

[16] A. Flores-Tlacuahuac, L.T. Biegler, E. Saldívar-Guerra. Dynamic Optimization of HIPS Open-Loop Unstable Polymerization Reactors. Ind.Eng.Chem.Res.,44:26592674,2005.

[17] J.P. Congalidis, J.R. Richards and W.H. Ray. AICHE J, 35 (1989) 891.

[18] R. Mahadevan, F.Doyle, A.C. Allcock, AIChE J, 48 (2002) 1754. 